Check for updates

Cite this: Phys. Chem. Chem. Phys., 2020, 22, 25538

Received 12th September 2020 Accepted 23rd October 2020

DOI: $10.1039 / d 0 c p 04825 a$

rsc.li/pccp

\section{Understanding benzyl alcohol aggregation by chiral modification: the pairing step $\dagger$}

\begin{abstract}
Robert Medel (D) and Martin A. Suhm (DD *
A combination of linear infrared and Raman spectroscopy in supersonic slit jet expansions is used to clarify the conformational preferences in the dimer of the transiently chiral benzyl alcohol (phenylmethanol) under vacuum isolation. By experimentally exploring close analogies with the permanently chiral 1-phenylethanol, which is conformationally locked in the jet through intramolecular chirality induction, and by computational analysis of their conformational energy landscapes, several conclusions are drawn. The lowest energy dimer is confirmed to be cooperatively $\mathrm{OH} \cdots \mathrm{OH} \cdots \pi$-bonded and shown to be homochiral. Its heterochiral counterpart is slightly higher in energy and can be spectrally assigned as a minor constituent. A metastable heterochiral $\mathrm{OH} \cdots \pi / \mathrm{OH} \cdots \pi$ structure with weakly coupled hydrogen bonds is efficiently trapped behind a $C_{i}$ symmetry-enhanced barrier and can be assigned by IR/Raman mutual exclusion. Its homochiral counterpart is kinetically less stable but might be addressed by rotational spectroscopy. Ratings of standard density functionals with a standard basis set in terms of reproducing these experimental chirality synchronization benchmarks are presented.
\end{abstract}

\section{Introduction}

When two entities of a transiently chiral molecule form a noncovalent dimer, they will have an energetical preference for either homo- (hom) or heterochiral (het) pairing - they synchronize their chirality in one way or the other. If this preference can be experimentally observed, it provides a sensitive test for the ability of quantum-chemical methods to describe subtle macroscopic phenomena such as Viedma ripening, ${ }^{1}$ chirality induced spin-selectivity, ${ }^{2}$ liquid crystal organization $^{3}$ or conglomerate crystallization in general. ${ }^{4}$ To reduce complexity, it makes sense to investigate the phenomenon of intermolecular chirality synchronization in the gas phase ${ }^{5}$ looking at small clusters. As the driving force for chirality synchronization in such a cluster can be quite small, low temperatures, as they are achievable in supersonic jet expansions, are indicated. However, at low temperature, the interconversion barriers between enantiomeric conformers quickly become insurmountable and statistical dimer formation prevails. $^{6}$ The relative energy information is then hidden on the experimental side. The goal is thus to find molecular systems where these barriers are sufficiently transmissive, such that subtle chirodiastaltic energies ${ }^{7}$ can still be

Institute of Physical Chemistry, University of Goettingen, Tammannstr. 6, 37077 Goettingen, Germany. E-mail: msuhm@gwdg.de

$\dagger$ Electronic supplementary information (ESI) available: Two-dimensional conformational scans of the monomers, more details and transition states of dimers, list of experimental band positions. See DOI: 10.1039/d0cp04825a detected and used for experimental benchmarking. Furthermore, one needs aggregation-sensitive chromophors for spectroscopic detection. In the field of vibrational spectroscopy, the $\mathrm{OH}$ group fulfills both requirements due to its partially tunneling-assisted conformational flexibility and pronounced hydrogen bond shifts. Elementary alcoholic examples of chirality synchronization are the dimers of ethanol, ${ }^{8,9}$ of trifluoroethanol, ${ }^{10,11}$ and of benzyl alcohol. ${ }^{12,13}$ Benzyl alcohol (B, Fig. 1) is among the most interesting. transiently chiral alcohols. In contrast to ethanol, ${ }^{14}$ it clearly favors a chiral ground state conformation. This actually involves the intramolecular synchronization of two adjacent, strongly coupled conformational degrees of freedom (circular arrows in Fig. 1). In contrast to trifluoroethanol, B offers alternative aggregation into symmetric dimers, because its $\pi$ system can compete with the $\mathrm{OH}$ group as a hydrogen bond acceptor. Depending on the relative chirality of the monomers, $C_{2}$ or $C_{\mathrm{i}}$ symmetry can be realized in the hom and het dimers, respectively. These symmetrical aggregates compete with unsymmetric complexes on the basis of $\mathrm{OH} \cdots \mathrm{OH}$

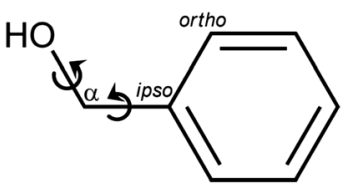

benzyl alcohol (B)

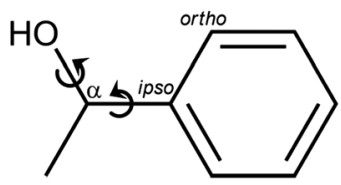

1-phenylethanol (P)
Fig. 1 Two-dimensional structural formulas, names, abbrevations, atom labeling and main conformational degrees of freedoms of the molecules investigated in this work. 
hydrogen bonds. This question of symmetry for dimers of $\mathbf{B}$ has already been discussed 80 years ago ${ }^{15}$ based on solution phase IR measurements. The spectral distinction of these dimer structures in the present work profits from the combination of IR and Raman spectroscopy in supersonic jets, also exploiting the rule of mutual exclusion.

Aromatic alcohols allow for a large repertoire of vibrational spectroscopy techniques, with size- and conformationally selective UV/IR double resonance techniques featuring prominently. Indeed, a dimer of $\mathbf{B}$ was observed two decades ago ${ }^{16,17}$ using such techniques and identified after correction ${ }^{12}$ of an initial size assignment. Chirality synchronization was not discussed at the time, but the spectra were illustrated with unsymmetric het dimers. $^{12}$

Here, we use linear FTIR spectroscopy and spontaneous Raman scattering, which offer nicely complementary viewpoints on the dimer as well as relative abundances of different isomers. Their disadvantage of reduced sensitivity is compensated by long, pulsed slit nozzles $\left(\mathrm{FTIR}^{6,18}\right)$ and powerful lasers $\left(\right.$ Raman $\left.^{6}\right)$. The lack of size selectivity is compensated by concentration variation and conformational discrimination is facilitated by the sensitivity of the $\mathrm{OH}$ chromophore to subtle interaction details. Our study builds on a preliminary investigation, which only involved FTIR spectroscopy ${ }^{13}$ and which implied unsymmetric hom dimers for the dominant signals from the UV/IR studies, although the het counterparts were only predicted slightly higher in energy and similar in their spectra. ${ }^{19}$ At the time, evidently neither UV/IR nor FTIR studies were able to rigorously discriminate between unsymmetric hom and het dimers of $\mathbf{B}$. A new finding of the FTIR study ${ }^{13}$ was the observation of a less downshifted $\mathrm{OH}$ stretching band, which was attributed to a symmetric dimer. Again, the relative chirality and thus the $C_{2}$ or $C_{\mathrm{i}}$ symmetry of this dimer had to remain open.

The dimer conformational landscape of benzyl alcohol is the main goal of the present contribution. It is considerably more complex than that of phenol dimer. ${ }^{20}$ To disentangle its details, it is advantageous to chemically freeze the transient chirality of B without introducing major perturbations in the dimer contact areas. This leads to 1-phenylethanol (P, Fig. 1), which is a wellstudied component of mixed dimers, ${ }^{21-28}$ whereas its selfaggregation appears to have remained unexplored in supersonic jet expansions. The extra methyl group, which introduces a permanent chirality center in $\mathrm{C}_{\alpha}$-position, leads to subtle but systematic modulations in the dimer energy landscape and allows to distinguish hom from het dimers. This strengthens the conclusions about benzyl alcohol dimerization and reveals chirality-sensitive relaxation paths during the dimerization process. Such chirality-dependent trapping of metastable isomers could be of mechanistic relevance for kinetically controlled enantioselective processes, also in solution. However, an experimental and computational study of $\mathbf{P}$ in solution suggests that the condensed phase is not the right starting point, even more so if dispersion corrections are neglected. ${ }^{29}$ This will become evident from the present results.

Beyond the question of whether hom or het pairings are preferred in the dimers of $\mathbf{B}$ and $\mathbf{P}$ and which hydrogen bond topology they exhibit, the lowering or raising of the racemization barrier in $\mathbf{B}$ by dimerization will be investigated. In the analogous aggregation study of transiently chiral 1,2-ethanediol and its chirality-controllable 1,2-cyclohexanediol homolog, ${ }^{6}$ the barrier was found to be lowered significantly, ${ }^{30}$ but not enough to prevent largely statistical hom/het dimer formation in a jet expansion. In monomeric $\mathbf{B}$, the barrier is an order of magnitude lower, providing a better starting point for chirality synchronization. In combination with its conformational uniformity at low temperatures, the dimerization process of $\mathbf{B}$ is thus a rewarding research objective.

Spectroscopic evidence for chirality-sensitive $\mathbf{B}$ and $\mathbf{P}$ oligomerization beyond pairing will only be addressed briefly to discriminate against dimer signals. Ultimately, such oligomers can be further discussed in the context of conglomerate formation for macroscopic B crystals, ${ }^{31}$ which consist of homochiral chains of cooperative $\mathrm{OH}$..O hydrogen bonds. The subtlety of this preference is illustrated by the thiol homolog of $\mathbf{B}$, where heterochiral dimer units with two isolated $\mathrm{SH} \cdots \pi$ contacts are present. ${ }^{31}$ The crystal structure and ee-dependent phase diagram of $\mathbf{P}$ is so far unknown, but would be of considerable interest. Is it a conglomerate former like B? Here, we provide the most elementary molecular entry point for the understanding of such macroscopic self-organisation phenomena.

\section{Methods}

\subsection{Experimental techniques}

At $24{ }^{\circ} \mathrm{C}$ a gas mixture with estimated molar fractions of $0.02 \%$ of either racemic $(98.5 \%$, Fluka) or enantiopure $\mathbf{P}(99.6 \%$, 99.6\% ee, (-)-(S)-enantiomer, Sigma Aldrich) in helium (99.996\%, Linde) was prepared and expanded at 1.20 bar and room temperature through a $(600 \times 0.2) \mathrm{mm}^{2}$ slit nozzle ${ }^{18}$ into vacuum. The pulsed supersonic expansion was probed by about 500 (racemic) or 200 (enantiopure) averaged synchronized FTIR scans at $2 \mathrm{~cm}^{-1}$ resolution with a Bruker IFS $66 \mathrm{v} / \mathrm{S}$ spectrometer (single-sided-fast-return mode). For the less volatile $\mathbf{B}$ (99\%, Alfa Aesar) a different heated setup was used with the compound at $70{ }^{\circ} \mathrm{C}$ (estimated molar fraction of $0.2 \%$ in $\mathrm{He}$ ). The pulsed expansion of the mixture at 1.5 bar through a slightly angled $(60 \times 0.2) \mathrm{mm}^{2}$ slit nozzle ${ }^{6}$ at $90{ }^{\circ} \mathrm{C}$ was probed by about 300 averaged synchronized FTIR scans at $2 \mathrm{~cm}^{-1}$ resolution with a Bruker IFS $66 \mathrm{v} / \mathrm{S}$ spectrometer (doublesided-fast-return mode).

For the Raman spectra ${ }^{32}$ of $\mathbf{B}$, as well as of enantiopure and racemic $\mathbf{P}$ (99.5\%, Aldrich), gas mixtures were prepared with the compounds at $50{ }^{\circ} \mathrm{C}$ (estimated $0.05 \%$ and $0.13 \%$ molar fractions in He for $\mathbf{B}$ und $\mathbf{P}$, respectively). The continuous expansion of the gas mixture at 1.0 bar through a $(4 \times 0.15) \mathrm{mm}^{2}$ slit nozzle at $60{ }^{\circ} \mathrm{C}$ was probed at $2 \mathrm{~mm}$ distance by a Spectra Physics Millenia eV laser (532 nm, cw, $25 \mathrm{~W}$ ). The scattered light was collected perpendicular to the laser and the nozzle flow via a camera lens and focused onto a one meter monochromator (McPherson). Photons from Stokes Raman scattering were co-added by a $\mathrm{LN}_{2}$-cooled CCD-camera (Princeton, PyLoN 400) 
over 3 min exposure time and averaged over 20 repetitions. The combination of laser and monochromator results in a resolution of about $1 \mathrm{~cm}^{-1}$. The spectra were calibrated with neon vacuum transitions. We assume band positions to be accurate up to $\pm 1 \mathrm{~cm}^{-1}$ and their differences up to $\pm 2 \mathrm{~cm}^{-1}$ in both FTIR and Raman spectra. The absence of optical rotation for the racemic samples was checked by polarimetry.

\subsection{Computational techniques}

All computations were carried out with the Gaussian 09 Rev. E. $01^{33}$ program package, except those for B2PLYP-D3 for which due to technical reasons partly also Gaussian 16 Rev. A.03 was used (the agreement of the results from both program versions was checked). Initial searches for minima and transitions states were conducted with the $\mathrm{B}_{3} \mathrm{LYP}^{34-37}$ functional together with Grimmes D3 correction with two-body terms and Becke-Johnson damping ${ }^{38}$ and the def2-TZVP basis set. ${ }^{39}$ This choice is based on previous performance for systems with chirality recognition. ${ }^{6,28}$ Results were reoptimized with the larger minimally augmented may-cc-pVTZ basis set $^{40}$ and complemented by B2PLYP-D3 ${ }^{41}$ (also with two-body terms and BJ-damping), $\omega \mathrm{B} 97 \mathrm{X}-\mathrm{D}^{42}$ and M06- $2 \mathrm{X}^{43}$ functional results, also using may-ccpVTZ. Specification of the basis set is therefore omitted hereafter. The ultrafine integration grid and verytight convergence criteria for monomers respectively tight for dimers were used. No density fitting was applied. The Hessian was evaluated within the double-harmonic approximation. Minima were confirmed by the absence, and transition states by the presence of exactly one imaginary frequency. The respective negative curvature coordinate was visualized to confirm the character of the transition states. The calculation of Raman activities at B2PLYP-D3 level proved to be unaffordable for systems of this size. All used keywords are listed in the ESI. $\dagger$

The dimer conformations reported in this work build on previous work ${ }^{13}$ for $\mathbf{B}$ and are based on systematic and extensive manual searches which are expected to capture all relevant structures for a supersonic jet expansion and many more which will only contribute at higher temperatures.

\subsection{Comparison between experiment and theory}

While the applied harmonic approximation strongly overestimates wavenumbers of $\mathrm{OH}$ stretching transitions, a better agreement with experiment can be achieved if differences between transitions for hydroxy groups in similar environments are compared. The neglected anharmonicity and other systematic errors are then expected to largely cancel out, especially if only the relative chirality of the involved molecules in a dimer motif is switched, as we have explored in a previous study. ${ }^{28}$ With a reliable anharmonic treatment out of reach for this system size, comparing data for related systems helps to understand whether deviations are systematic or erratic for assignment purposes.

The unsymmetric dimers of $\mathbf{B}$ and $\mathbf{P}$ feature two hydroxy groups involved in hydrogen bonds, with a $\pi$ and an oxygen acceptor, respectively. These lead to two transitions in the $\mathrm{OH}$ stretching region with substantial IR as well as Raman activity, whose measured relative intensities can be compared with predictions.
Experimental relative intensities and their uncertainties are evaluated by integration with a Monte Carlo approach ${ }^{44}$ which accounts for ambiguity from integration width and noise level.

Theoretical IR band strengths were taken directly from the computational results, while calculated Raman activities and depolarisation ratios were converted to scattering cross sections. Accounted for are the polarisation dependent sensitivity of monochromator and camera, the laser wavelength and partial thermal vibrational excitation. This is detailed in the ESI. $\dagger$

Relative band intensities from different conformers are in addition dependent on their population ratio and thus, assuming a Boltzmann distribution, strongly on their energy difference and the conformational freezing temperature ${ }^{45} T_{\mathrm{c}}$. The latter is in a supersonic expansion partially barrier dependent and can only be roughly estimated, unless ${ }^{8}$ the energy gap is experimentally well determined by other means. ${ }^{14}$ Calculation of this quantity by eqn (1) combines experimental intensity ratios $I_{i} / I_{j}$ for two isomers $i$ and $j$ with the computed vibrational zeropoint corrected energy difference $E_{j}^{0}-E_{i}^{0}$, theoretical IR band strength or Raman cross section ratios $\sigma_{j} / \sigma_{i}$, and ratios of chiral degeneracies $g_{j} / g_{i}$ as well as of rotational symmetry numbers $s_{i} / s_{j}$.

$$
T_{\mathrm{c}}=\frac{E_{j}^{0}-E_{i}^{0}}{R}\left[\ln \left(\frac{I_{i}}{I_{j}} \cdot \frac{\sigma_{j}}{\sigma_{i}} \cdot \frac{g_{j}}{g_{i}} \cdot \frac{s_{i}}{s_{j}}\right)\right]^{-1}
$$

This assumes that the rotational and vibrational partition functions of the two isomers are similar in the cold jet expansion. For a shallow isomerization barrier, one expects conformational temperatures between about $20 \mathrm{~K}$ (close to the rotational temperature in a slit jet expansion) and about $100 \mathrm{~K} .^{8,46,47}$ Values between 0 and $20 \mathrm{~K}$ indicate an underestimation of the energy difference. For high-barrier inter-monomer isomerizations, $T_{\mathrm{c}}$ can be elevated up to the nozzle temperature. Larger values are unexpected, as are negative $T_{\mathrm{c}}$-values, with the latter being equivalent to a wrongly predicted energy sequence. These values are thus a measure of the quality of the predicted energy difference, but also of the size of the barriers separating the isomers. Due to differences in the experimental setups described before, in particular in terms of probed expansion zone, ${ }^{8}$ some variance in the conformational temperatures between IR and Raman measurements is expected. The range of mean values obtained from $100000 \mathrm{MC}$ evaluations of the relative band intensities for up to four band pairs will be discussed here in the main document. Separate values for the evaluation of each band pair as well as 95\% confidence intervals from $2.5 \%$ and $97.5 \%$ quantiles as error bars are available in Fig. S10-S13 in the ESI. $\dagger$

The performance of the employed harmonic DFT methods to predict the relative energy, $\mathrm{OH}$ stretching wavenumbers and spectral visibility of isomers will be summarized in Fig. 12 in the conclusion.

\section{Results and discussion}

\subsection{Computational results}

3.1.1 Monomer conformations. The main conformational degrees of freedom of both $\mathbf{B}$ and $\mathbf{P}$ can be described by two 
dihedral angles: $\mathrm{OC}_{\alpha} \mathrm{C}_{i p s o} \mathrm{C}_{\text {ortho }}$ and $\mathrm{HOC}_{\alpha} \mathrm{C}_{\text {ipso }}$, which can be modified by rotation around the $\mathrm{C}_{\alpha}-\mathrm{C}_{i p s o}$ and $\mathrm{O}-\mathrm{C}_{\alpha}$ bonds, respectively, as highlighted in Fig. 1. For the former heavyatom dihedral we classify the qualitative arrangement with a capital letter (e.g. $G+$ for gauche with a value of roughly $+60^{\circ}$ ), for the latter dihedral we use a lower case letter instead. Full range two-dimensional relaxed scans are available in Fig. S3 and S6 in the ESI. $\dagger$ From the two ortho carbon atoms we choose here the closest one for the determination of the heavy-atom dihedral, so that it is constrained between $-90^{\circ}$ and $+90^{\circ}$. The global minima of $\mathbf{B}$ are two mirror-image conformers with both dihedrals synchronized in gauche arrangements with the same sign: $G-g-$ and $G+g+$ (Fig. 2), in agreement with previous calculations $^{13,48-52}$ and results from rotational spectroscopy. ${ }^{51}$ They can interconvert via a shallow barrier of $2.0 \mathrm{~kJ} \mathrm{~mol}^{-1}$ (B3LYP-D3, zero-point corrected), even down to low conformational temperatures in a jet expansion. This interconversion is supported by heavy-atom tunneling in the vibrational ground state with a frequency of about $500 \mathrm{MHz},{ }^{51}$ equivalent to significant interference between the two localized structures in the torsional eigenstates. This has attracted considerable interest in recent years for $\mathbf{B}$ and its derivatives. ${ }^{51-58}$ The gas phase conformation resembles the one in the crystal structure. ${ }^{31}$ Another conformer is predicted with an eclipsed $(E)$ respectively trans $(t)$ arrangement of the dihedrals, with all heavy atoms and the hydroxy hydrogen (almost) in the plane of the phenyl ring. However, with a predicted relative energy of about $4 \mathrm{~kJ} \mathrm{~mol}^{-1}$ and a similarly shallow interconversion barrier it is not observed in jet expansions ${ }^{12,13,50,51}$ and even remains elusive at room temperature. ${ }^{50}$

The additional methyl group at the $\alpha$-carbon in $\mathbf{P}$ acts as a conformational anchor for the relative stabilization of one of

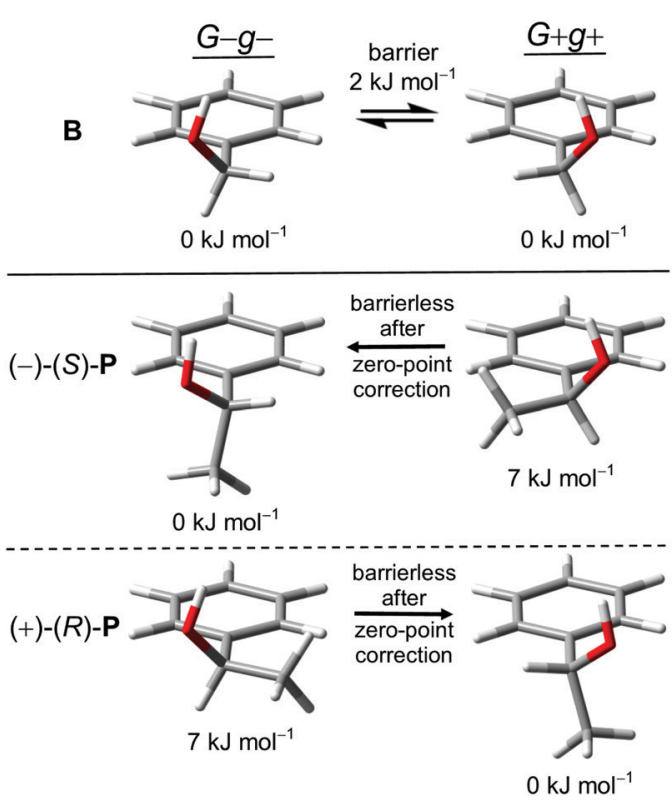

Fig. 2 G-g- and $G+g+$ monomer conformers of B, (-)-(S)-P and $(+)-(R)-\mathbf{P}$ with relative zero-point corrected energies and interconversion barriers at B3LYP-D3 level. the $G g$ conformations depending on the chirality of the stereogenic center: $G-g-$ for $(-)-(S)-\mathbf{P}$ and $G+g+$ for $(+)-(R)-P$. In comparison to $\mathbf{B}$ the absolute value of the $\mathrm{OC}_{\alpha} \mathrm{C}_{i p s o} \mathrm{C}_{\text {ortho }}{ }^{-}$ dihedral is predicted to be slightly lowered (from $55^{\circ}$ to $43^{\circ}$ ) due to competition with the preference of the methyl group for a position perpendicular to the plane of the phenyl ring, as observed in ethylbenzene. ${ }^{59}$ In contrast, the other $G g$ arrangement with the signs of the dihedrals opposite to the standard optical rotation of the enantiomer is moved up in energy by about $7 \mathrm{~kJ} \mathrm{~mol}^{-1}$ due to a far less favorable positioning of the methyl group relative to the phenyl ring (Fig. 2). This is a case of intramolecular chirality induction. ${ }^{5}$

Besides a high energy Et-conformer similar to B, we located two additional minima on the electronic potential surface which were not mentioned in previous computational investigations of $\mathbf{P} .^{24,29,49,60,61}$ They are also very high in energy (6-7 $\mathrm{kJ} \mathrm{mol}^{-1}$ ) and have vanishing relaxation barriers after vibrational zero-point correction. Structures and properties of the higher energetic conformers of $\mathbf{B}$ and $\mathbf{P}$ are given in the ESI. $\dagger \mathbf{P}$ is thus a rare case of a permanently chiral alcohol with one dominant conformer, while most occur in multiple energetically close conformations due to the low symmetry of the $\mathrm{HOC}_{\alpha} \mathrm{C}_{\beta}$ torsional potential. ${ }^{28,62}$

In conclusion, $\mathbf{B}$ is expected to occur as a 1:1-mixture of two rapidly interconverting transiently chiral conformers, whereas the ratio of the analogous permanently chiral conformers of $\mathbf{P}$ can be controlled by the enantiomeric excess of the sample.

3.1.2 Dimer conformations. The irrelevance of higher energy conformations in supersonic jet studies helps to keep the dimer nomenclature simple. We largely adopt and extend it from earlier $\mathbf{B}$ work $^{13}$ and focus on those elements which are necessary to describe the six most important dimer structures. For a list and extended nomenclature of higher lying structures, see the ESI. $\dagger$ The relative chirality of the constituents is encoded with hom/het and the hydrogen bond acceptors are denoted with $\mathrm{O}$ or $\pi$. The two electron lone pairs (E) of the oxygen atom in the most stable monomer conformation are diastereotopic and are classified with respect to the torsional angle $\mathrm{EOC}_{\alpha} \mathrm{C}_{\text {ipso }}$ as gauche $\left(\mathrm{O}^{g}\right)$ or trans $\left(\mathrm{O}^{t}\right)$. If there is a symmetry element in the structure, it is added for convenience.

The five most stable $\mathbf{B}$ dimer structures and the het equivalent to the least stable of them are shown and characterized in Fig. 3, with consistent configuration $(G-g-)$ and orientation of the $\mathrm{OH} \cdots \pi$ hydrogen bond donor to illustrate differences in the interaction with the second molecule. The pairwise hom/het analogy also applies to many higher-lying conformations described in the ESI $\dagger$ and confirms the suitability of this system for chirality synchronization studies. The predicted chirodiastaltic energy varies in sign and size among the motifs and is of the same order of magnitude as the racemization barrier.

The most stable structural motif $\mathrm{O}^{g} \pi$ features cooperatively interacting $\mathrm{OH} \cdots \mathrm{OH} \cdots \pi$ hydrogen bonds and is reminiscent of the global minimum of the propargyl alcohol dimer. ${ }^{63,64}$ The het variant is slightly less stable despite somewhat stronger hydrogen bonds (based on predicted downshifts and intensity enhancements), indicating a compensating role of 

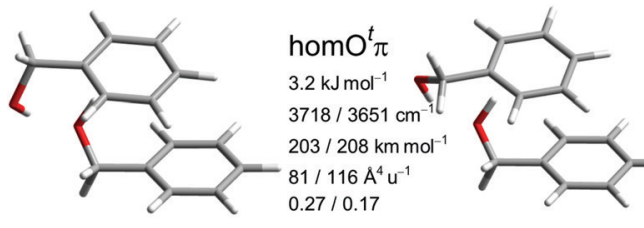

$\operatorname{hetO}^{t} \pi$

$4.8 \mathrm{~kJ} \mathrm{~mol}^{-1}$ $3720 / 3664 \mathrm{~cm}^{-1}$ $200 / 194 \mathrm{~km} \mathrm{~mol}^{-1}$ $74 / 121 \AA^{4} u^{-1}$ $0.27 / 0.17$
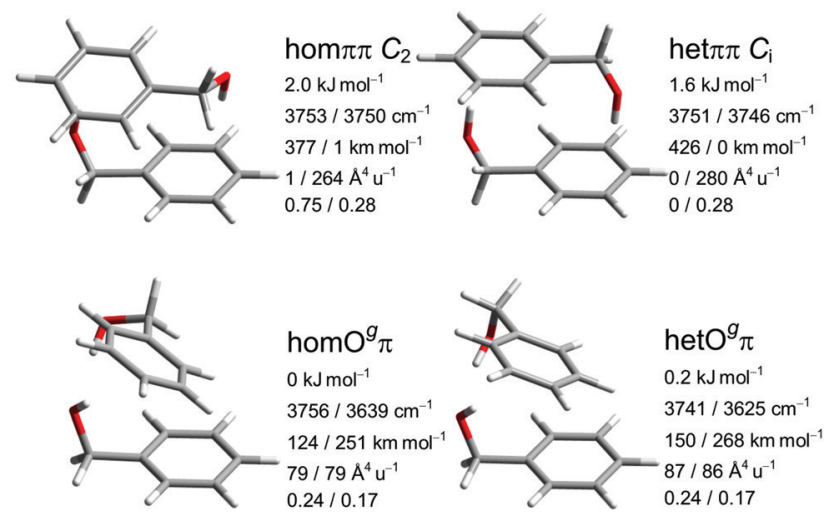

Fig. 3 Leading $\mathbf{B}$ dimer structures with harmonically zero-pointcorrected relative energies, unscaled harmonic $\mathrm{OH}$ stretching wavenumbers, their IR intensities, Raman activities and depolarization ratios obtained at B3LYP-D3 level.

dispersion interactions. The motif with the alternative lone pair coordination $\mathrm{O}^{t} \pi$ is not energetically competitive for $\mathbf{B}$ but still included, because it gains importance for $\mathbf{P}$ and helps to understand relaxation paths. $\mathrm{O}^{t} \pi$ offers a stronger $\mathrm{OH} \cdots \pi$ interaction at the expense of the $\mathrm{OH} \cdots \mathrm{O}$ hydrogen bond, judging by the downshift. The resulting smaller difference in wavenumber of the OH-oscillators leads to a limited coupling between them, transferring IR intensity to the higher-frequency (partially antisymmetric) and Raman intensity to the lowerfrequency (partially symmetric) mode.

The two unsymmetric $\mathrm{O} \pi$ motifs energetically sandwich the symmetric $\pi \pi$ motif with two isolated, non-cooperative $\mathrm{OH} \cdots \pi$ hydrogen bonds. Depending on the relative chirality of the monomers, a two-fold rotation axis (hom) or an inversion center (het) emerges and generates mutual (quasi-)exclusion

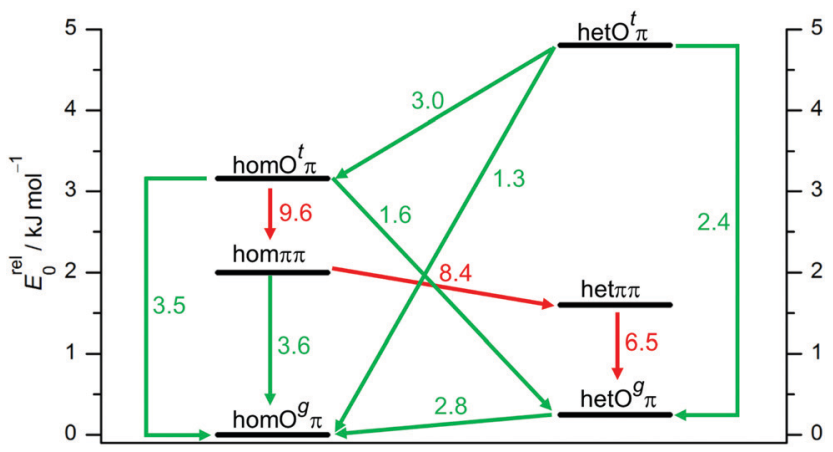

Fig. 4 Interconversion paths between $\mathbf{B}$ dimer structures including downhill barriers in $\mathrm{kJ} \mathrm{mol}^{-1}$ at B3LYP-D3 level which are more (green) or less (red) feasible under supersonic jet expansion conditions. Transition structures are given in the ESI. $\uparrow$ of IR and Raman intensity as well as a small (so called Davydov or vibrational exciton ${ }^{65}$ ) splitting between the two states which depends on the coupling between the degenerate monomer modes. Spectrally, this increases their visibility, whereas statistically (due to the symmetry number in the rotational partition function for hom, and achirality for het) and energetically the symmetric structures have a handicap. We note that the het $\pi \pi$ variant is realized in the crystal structure of the thiol analog ${ }^{31}$ of $\mathbf{B}$. In the gas phase, there are also cases where the symmetric dimer motif wins, such as lactates, ${ }^{66}$ glycidol $^{67}$ and glycolaldehyde. ${ }^{68}$ This is a consequence of the better acceptor strength of $O$ compared to $\pi$ in these molecules. It is already remarkable that $\pi \pi$ can be observed at all at low temperature in competition to $\mathrm{OH} \cdots \mathrm{OH} \cdots \pi$ arrangements for $\mathbf{B}^{13}$

To better understand the population of different hydrogen bonding motifs under jet conditions, it is important to analyze barriers separating them. Fig. 4 shows the connections between the three structural motifs for both relative chiralities. The indicated barrier heights refer to the downhill transition and include harmonic zero-point energy of all modes with real, positive wavenumber. As a rule of thumb, $4-5 \mathrm{~kJ} \mathrm{~mol}^{-1}$ represent a rough border between facile (green) and difficult (red) interconversion under supersonic jet conditions. ${ }^{69}$ We cannot strictly exclude lower, more complex interconversion paths involving concerted changes of hydrogen bond topology and transient chirality, but believe that the network depicted in Fig. 4 is representative.

The lone pair switch between the $\mathrm{O}^{t} \pi$ and $\mathrm{O}^{g} \pi$ motifs is predicted with moderate barriers of 2.4 and $3.5 \mathrm{~kJ} \mathrm{~mol}^{-1}$ and should be feasible for both transient diastereomers. A chirality switch at the acceptor molecule in an $\mathrm{OH} \cdots \mathrm{O}$ hydrogen bond interconverts the ${ }^{g}$ or ${ }^{t}$ character of its lone pairs. This coupled relative chirality and binding motif conversion only needs to overcome a barrier of 1.3 or $1.6 \mathrm{~kJ} \mathrm{~mol}^{-1}$, depending on whether it leads to or away from homochirality, respectively. Compared to the monomer value of $2.0 \mathrm{~kJ} \mathrm{~mol}^{-1}$, this corresponds to a certain enhancement of the transient character of chirality in $\mathbf{B}$ upon coordination of the $t$ lone pair. In a subtle sense, $\mathrm{O}^{t} \pi$ structures may be viewed as catalysts for chirality inversion. In contrast, the interconversion of a donor of an $\mathrm{OH} \cdots \mathrm{O}$ hydrogen bond is perhaps somewhat decelerated by an increased barrier in the $2.8-3.0 \mathrm{~kJ} \mathrm{~mol}^{-1}$ range (Fig. 4).

The $\pi \pi$ motif behaves very differently. It features a much higher hom-to-het interconversion barrier $\left(8.4 \mathrm{~kJ} \mathrm{~mol}^{-1}\right)$, because symmetric dimerization guides both absolute ${ }^{O} C_{\alpha} \mathrm{C}_{i p s o} \mathrm{C}_{\text {ortho }}$ backbone torsion angles further away from the $90^{\circ}$ transition state value, from the monomer value of $55^{\circ}$ to only $17^{\circ}$ for het $\pi \pi$ and to $31^{\circ}$ for hom $\pi \pi$, respectively. This adaptive aggregation is driven by the minimization of the distance between the two monomers, which is disrupted in the transition state and therefore discourages racemization. It also provides a geometrical explanation why het $\pi \pi$ is somewhat more stable than hom $\pi \pi$. An alternative racemization path with opposite torsion direction is already quite unattractive for the monomer and even more so for the symmetric dimers.

As direct chirality synchronization within the $\pi \pi$ motif is difficult under supersonic jet conditions, it is important to analyze the barriers separating them from $\mathrm{O} \pi$ structures. 
This requires an acceptor switch from the $\pi$ system of the phenyl ring to a lone pair of the oxygen and thus a rather pronounced relative rotation of the two monomers, to bring the two hydroxy groups into close neighborhood. This trajectory can be described by the $\mathrm{C}_{\alpha} \mathrm{C}_{i p s o} \mathrm{C}_{i p s o}^{\prime} \mathrm{C}_{\alpha}^{\prime}$ intermolecular torsion angle, which was systematically scanned to locate the transition states. Starting at $\operatorname{hom} \pi \pi$, the two scan directions are not equivalent. One of them ultimately leads downhill over a $3.6 \mathrm{~kJ} \mathrm{~mol}^{-1}$ barrier to homO ${ }^{g} \pi$, the other one uphill over a much higher $10.7 \mathrm{~kJ} \mathrm{~mol}^{-1}$ barrier involving a broken hydrogen bond, because the distance between the system and the targeted $\mathrm{O}^{t}$ lone pair is quite large. Basically, this means that hom $\pi \pi$ is expected to relax rather smoothly into the global minimum structure.

The situation for het $\pi \pi$ is different. Due to its inversion symmetry, both torsional directions are equivalent and lead to hetO $^{g} \pi$, over a barrier of $6.5 \mathrm{~kJ} \mathrm{~mol}^{-1}$. One possible explanation is that the donor $\mathrm{OC}_{\alpha} \mathrm{C}_{i p s o} \mathrm{C}_{\text {ortho }}$ torsion associated with the het isomerization spans a larger range $\left(17\right.$ to $\left.65^{\circ}\right)$ than in the hom case $\left(31\right.$ to $44^{\circ}$ ). In addition this trajectory also involves a larger torsion of both molecules with respect to each other along the $\mathrm{C}_{\alpha} \mathrm{C}_{i p s o} \mathrm{C}_{i p s o}^{\prime} \mathrm{C}_{\alpha}^{\prime}$ dihedral, which translates not only in a higher but also broader barrier. In any case, het $\pi \pi$ relaxation appears to be much more difficult than hom $\pi \pi$ relaxation.

In summary, all important $\mathbf{B}$ dimer structures are predicted to have feasible relaxation paths into lower energetic structures, except for het $\pi \pi$ and of course the global minimum homo ${ }^{g} \pi$. This will be important when interpreting the experimental spectra.

Trimers of $\mathbf{B}$ are beyond the scope of the present study, apart from the need to discriminate dimer signals in the nonselective linear spectra from trimer contributions. Here it suffices to note that they show interesting isomerism similar to that in 1-indanol trimers, ${ }^{70}$ namely reduced ring strain by switching from cyclic OOO motifs to $0 O \pi$ structures. This optional symmetry breaking leads to complex spectra which will be the topic of a separate study.

The close similarity of $\mathbf{P}$ dimers to their $\mathbf{B}$ counterparts (Fig. 3) is illustrated in Fig. 5, which lists the six most stable conformations together with their most important $\mathrm{OH}$ vibrational properties. Higher energy structures and their nomenclature are discussed in the ESI. $\dagger$

This similarity results from the fact that in all six cases the additional methyl group points away from the contact zone between the monomers. Indirect effects of this methyl group on the intramolecular torsion potential, on the polarization of the $\mathrm{OH}$ group and on the total dispersion interaction lead to modulations of the energetical spacings. The permanent chirality center introduced by this methyl group blocks the interconversion between the most stable hom and het dimers, because it effectively anchors the preferred handedness of the transient chirality elements which $\mathbf{P}$ shares with $\mathbf{B}$, as explained in the preceding subsection. Therefore, the enantiomeric composition of $\mathbf{P}$ controls the abundance of hetO ${ }^{g} \pi$ through intramolecular chirality induction without the risk of relaxing to homO $^{g} \pi$. This allows for hom/het assignments of $\mathbf{P}$ dimer signals which can be transferred to $\mathbf{B}$ with the help of harmonic
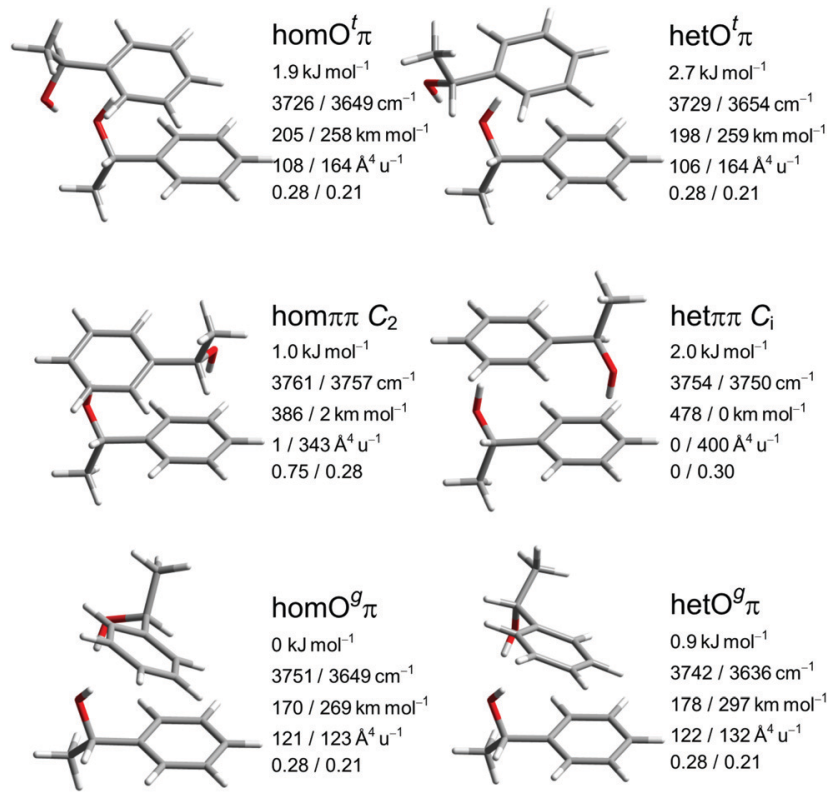

Fig. 5 Leading $\mathbf{P}$ dimer structures with harmonically zero-pointcorrected relative energies, unscaled harmonic $\mathrm{OH}$ stretching wavenumbers, their IR intensities, Raman activities and depolarization ratios obtained at B3LYP-D3 level.

spectral predictions, because theoretical deficiencies such as neglected anharmonicity should largely cancel between $\mathbf{P}$ and B. The same strategy can be used to spectrally differentiate hom $\pi$ from het $\pi \pi$. In terms of hom/het chirodiastaltic energy, the three most stable $\mathbf{P}$ dimer motifs have a clear preference for the hom pairing. Compared to $\mathbf{B}$ this means a switch in the energetic order of hom- and het $\pi \pi$, which can be attributed to the smaller intramolecular flexibility of $\mathbf{P}$ in the $\mathrm{OC}_{\alpha} \mathrm{C}_{i p s o} \mathrm{C}_{\text {ortho }}$ dihedral. This dihedral is especially far from its monomeric value in het $\pi \pi$ as discussed before for B. Our spectroscopic techniques do not allow for an experimental verification of this energetic prediction due to the blocked interconversion. This would require experimental bond dissociation measurements. ${ }^{71,72}$ Another difference between $\mathbf{P}$ and $\mathbf{B}$ dimers is the significant energetical lowering of the $\mathrm{O}^{t} \pi$ motif for $\mathbf{P}$, which is founded in comparable little deformation needed for the $\mathbf{P}$ monomer to adapt for this motif. This might allow detection in a supersonic jet expansion.

The lowest predicted interconversion barriers for $\mathbf{P}$ dimers are shown in Fig. 6. Again, there is a close analogy to B. Despite the blocked hom/het interconversion options, all excited conformers but het $\pi \pi$ are predicted to have feasible relaxation options to lower structures. This rather close parallel between predicted dimerization preferences for $\mathbf{B}$ and $\mathbf{P}$ sets the stage for a consistent interpretation of their dimer signals in supersonic jet spectra.

\subsection{Experimental results}

3.2.1 Experimental results for benzyl alcohol. The upper part of Fig. 7 provides two survey FTIR spectra of the $\mathrm{OH}$-stretching region for heated $\mathbf{B}$ expansions in helium. The difference reflects 


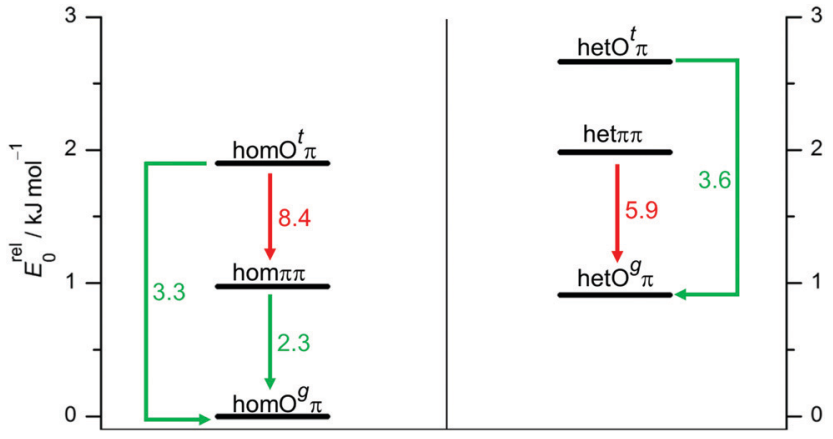

Fig. 6 Interconversion paths between $\mathbf{P}$ dimer structures including downhill barriers in $\mathrm{kJ} \mathrm{mol}^{-1}$ at B3LYP-D3 level which are more (green) or less (red) feasible under supersonic jet expansion conditions.

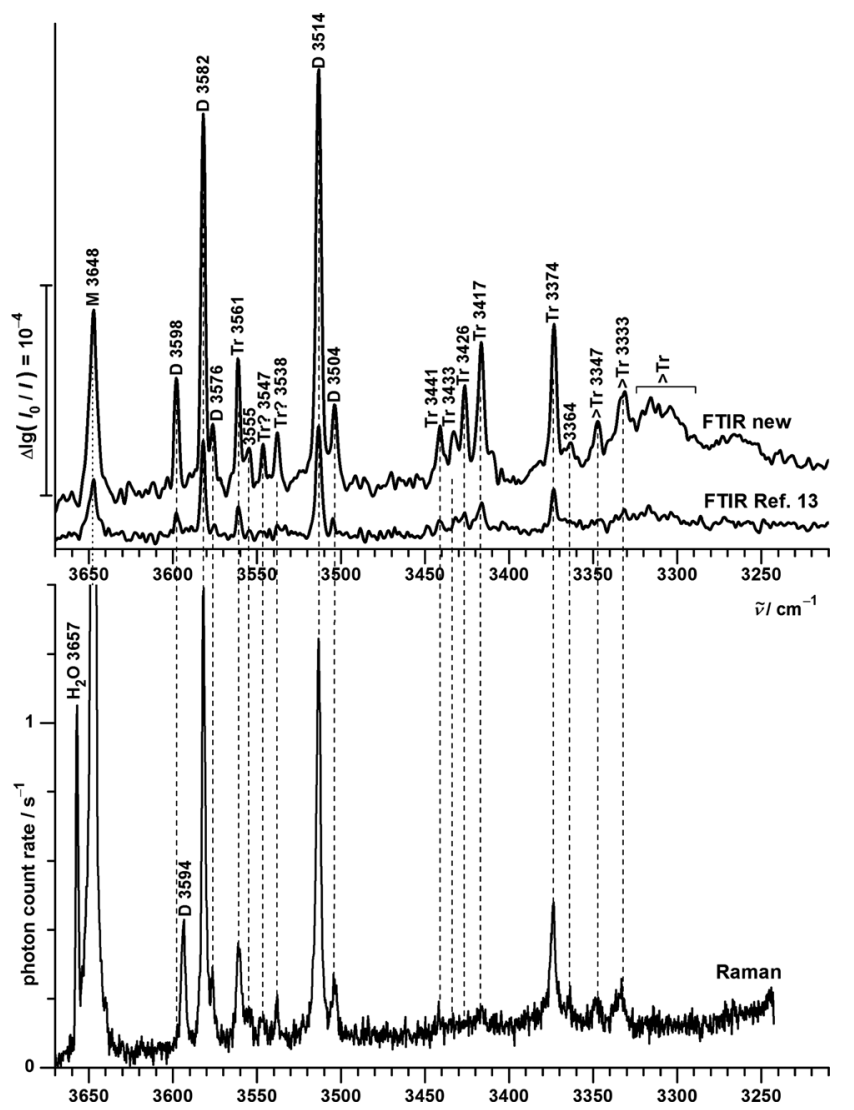

Fig. $7 \mathrm{OH}$ stretching spectra of $\mathbf{B}$ seeded in helium from FTIR (upper traces, comparing new ${ }^{73}$ (courtesy of M. Lange and E. Sennert) to published measurements ${ }^{13}$ ) and Raman (lowest trace, this work) spectroscopy. Cluster size assignments $(M=$ monomers, $D=$ dimers, $\operatorname{Tr}=$ trimers, $>\operatorname{Tr}=$ larger clusters according to previous ${ }^{13}$ and this work) are labeled together with the wavenumbers of the band maxima.

the signal-to-noise improvements achieved since the original $\mathbf{B}$ study, ${ }^{13}$ based on technical modifications which were recently described. ${ }^{6}$ The new spectrum (top trace) was obtained in the context of unpublished chirality induction studies ${ }^{73}$ and is kindly provided by M. Lange and E. Sennert. Nominally unchanged expansion parameters such as the composition of the gas mixture and the nozzle temperature lead to similar cluster distributions

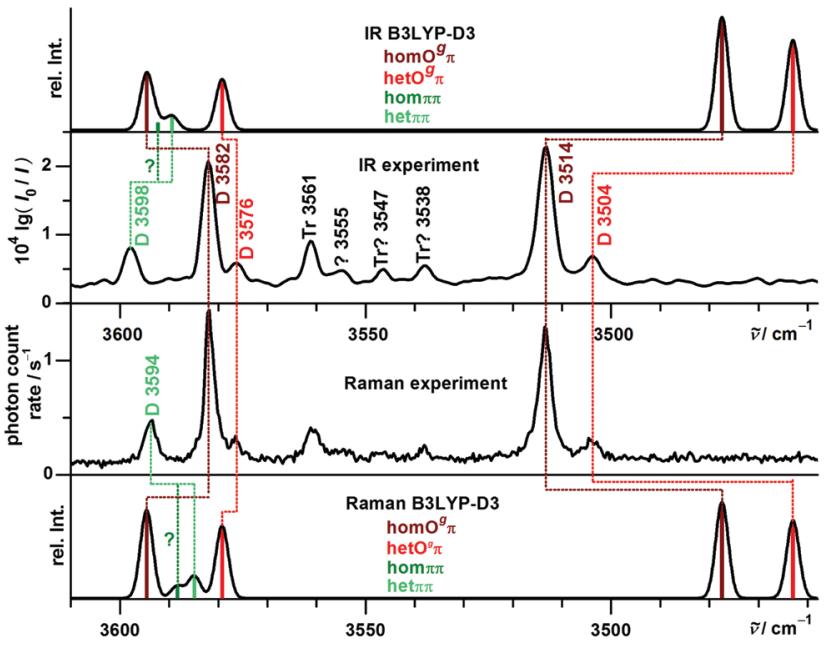

Fig. 8 B dimer B3LYP-D3 simulations (outer) compared to experimental IR and Raman jet spectra (inner traces) after shifting the harmonic $\mathrm{OH}$ stretching wavenumbers by $-161.5 \mathrm{~cm}^{-1}$ to match the monomer transition. A uniform conformational temperature of $100 \mathrm{~K}$ and a Gaussian FWHM of $3 \mathrm{~cm}^{-1}$ are assumed.

with $\mathrm{a} \approx 4$-fold signal gain due to an improved pulsed nozzle design. Size assignments are taken from concentration and temperature variation studies reported earlier ${ }^{13}$ and are complemented in this work.

The lowest trace shows a new heated nozzle Raman spectrum recorded $2 \mathrm{~mm}$ downstream from the nozzle, which also reveals cluster signals. Not yet reported bands common to the FTIR and Raman spectra are found at 3576, 3555 and $3364 \mathrm{~cm}^{-1}$. The band at $3504 \mathrm{~cm}^{-1}$ was previously interpreted cautiously because of overlap with a possible impurity, ${ }^{13}$ but is now seen to be also due to $\mathbf{B}$ dimers.

Most importantly, a Raman band without FTIR counterpart is observed at $3594 \mathrm{~cm}^{-1}$, whereas the FTIR band at $3598 \mathrm{~cm}^{-1}$ is completely missing in the Raman trace. This proves the previous tentative assignment of the FTIR band to a symmetric $\pi \pi$ dimer. ${ }^{13}$ The two bands with complementary IR and Raman intensity are Davydov-split by $4 \mathrm{~cm}^{-1}$ and they are quite narrow, such that a dominant assignment to either hom- or het $\pi \pi$ dimer is more likely than an accidental double coincidence.

Bands marked $\operatorname{Tr}$ and $>\operatorname{Tr}$ due to their different scaling behaviour do not behave systematically in the IR/Raman comparison. Some are significant in both spectral traces, others (in particular around $3430 \mathrm{~cm}^{-1}$ ) are very weak in the Raman spectrum, pointing at some symmetry effects. However, a closer analysis and extension of the frequency range will be needed for a detailed oligomer assignment and the following discussion thus focuses on the dimer range spanning $3600-3500 \mathrm{~cm}^{-1}$.

Fig. 8 compares the experimental spectra with harmonic B3LYP-D3 simulations, which are uniformly shifted to match the B monomer transition. Any deviations must be blamed on deficiencies of the density functional and on changes in the anharmonicity of the $\mathrm{OH}$ stretching mode upon aggregation.

The experimental Davydov splitting of $4 \mathrm{~cm}^{-1}$ between the $3598 / 3594 \mathrm{~cm}^{-1}$ bands would be consistent with predictions for 
Table 1 B hom $\pi \pi$ - het $\pi \pi$ dimer energy differences $\Delta E_{0}$ and hydrogen bonded $\mathrm{OH}$ downshifts $-\Delta \tilde{\nu}(\omega)$ relative to the $\mathrm{Gg}$ monomer at different harmonic levels in comparison with experiment

\begin{tabular}{lccc}
\hline Method & $\Delta E_{0} / \mathrm{kJ} \mathrm{mol}^{-1}$ & $-\Delta \tilde{\nu}(\omega) \mathrm{hom} / \mathrm{cm}^{-1}$ & $-\Delta \tilde{\nu}(\omega)$ het $/ \mathrm{cm}^{-1}$ \\
\hline Experiment & $?$ & & $50(2) / 54(2)$ \\
B3LYP-D3 & 0.4 & $56 / 59$ & $58 / 63$ \\
B2PLYP-D3 & 0.3 & $55 / 59$ & $56 / 60$ \\
$\omega$ B97X-D & 1.4 & $45 / 49$ & $47 / 51$ \\
M06-2X & -0.5 & $31 / 35$ & $30 / 35$
\end{tabular}

hom $\pi \pi\left(4.0 \mathrm{~cm}^{-1}\right)$ as well as het $\pi \pi\left(4.6 \mathrm{~cm}^{-1}\right)$. We favor the het dimer as the main contributor, because it is predominantly predicted to be more stable (except by the M06-2X prediction, see Table 1) and slightly more visible, and most importantly more hindered from relaxation by a significantly larger barrier. This will be confirmed later on by the experimental $\mathbf{P}$ analogy. It is typical that the underlying isolated $\mathrm{OH} \cdots \pi$ downshifts are slightly overestimated by harmonic B3LYP-D3 calculations (Table 1).$^{74}$ Whether there is also a minor overlapping contribution by hom $\pi \pi$ surviving the expansion might be resolvable by rotational spectroscopy (predicted dipole moment of $2 \mathrm{D}$ ), while het $\pi \pi$ is transparent due to the missing dipole moment.

With R2PI spectroscopy a number of signals were reported in the dimer mass channel, but due to spectral congestion only one conformer could be analyzed in the IR region with absorption at 3584 and $3515 \mathrm{~cm}^{-1} .{ }^{12}$ These signals are confirmed in this work at 3582 and $3514 \mathrm{~cm}^{-1}$ in both FTIR and Raman spectra. Assignment to one of the unsymmetric $\mathbf{B}$ dimers is not unambiguous. The single strong pair of bands is in contrast to the B3LYP-D3 and B2PLYP-D3 predictions for the hom- und hetO $^{g} \pi$ dimers to be nearly isoenergetic (Table 2). For the predicted low barrier, there should be an equilibrium of both structures in the jet expansion down to low temperature. Most likely, the energy difference is actually larger, because none of the calculations predicts a perfect spectral overlap for the two isomers. All investigated computational methods agree in predicting homO ${ }^{g} \pi$ to be more stable and less downshifted than hetO $^{g} \pi$. Therefore, the strong pair of bands is assigned to homO $\pi$ and one expects smaller satellites due to heto ${ }^{g} \pi$ towards lower wavenumber. One candidate at $3504 \mathrm{~cm}^{-1}$ has been postulated before ${ }^{19}$ and would match the theoretical predictions for the $\mathrm{OH} \cdots \mathrm{O}$ vibration acceptably well (Table 2). For the $\mathrm{OH} \cdots \pi$ component, we propose the new satellite band at $3576 \mathrm{~cm}^{-1}$, although the hom - het shift is predicted to be larger than the one for the $\mathrm{OH} \cdots \mathrm{O}$ mode (Table 2). Therefore, alternative assignments such as overlap with the trimer band at

Table 2 B hom $O^{g} \pi-$ het $O^{g} \pi$ dimer energy $\Delta E_{0}$ and $\mathrm{OH}$ stretching wavenumber differences $\Delta \tilde{\nu}(\omega)$ at different harmonic levels in comparison with experiment

\begin{tabular}{llll}
\hline Method & $\Delta E_{0} / \mathrm{kJ} \mathrm{mol}^{-1}$ & $\Delta \tilde{\nu}(\omega) \mathrm{OH} \cdots \pi / \mathrm{cm}^{-1}$ & $\Delta \tilde{\nu}(\omega) \mathrm{OH} \cdots \mathrm{O} / \mathrm{cm}^{-1}$ \\
\hline Experiment & $<0$ & $6(2)$ & $10(2)$ \\
B3LYP-D3 & -0.2 & 15 & 14 \\
B2PLYP-D3 & -0.1 & 16 & 14 \\
$\omega$ B97X-D & -1.7 & 18 & 8 \\
M06-2X & -2.8 & 7 & 6
\end{tabular}

Table 3 B hom $\mathrm{O}^{g} \pi$-het $\pi \pi$ energy $\Delta E_{0}$ and $\mathrm{OH}$ stretching wavenumber differences $\Delta \tilde{\nu}(\omega)$ (between the $\mathrm{OH} \cdots \pi$ mode of homO ${ }^{g} \pi$ and the symmetric or antisymmetric $\mathrm{OH}$ stretching mode of het $\pi \pi$, respectively) at different harmonic levels in comparison with experiment

\begin{tabular}{llll}
\hline Method & $\Delta E_{0} / \mathrm{kJ} \mathrm{mol}^{-1}$ & $\Delta \tilde{\nu}(\omega)$ antis. $/ \mathrm{cm}^{-1}$ & $\Delta \tilde{\nu}(\omega) \mathrm{symm} . / \mathrm{cm}^{-1}$ \\
\hline Experiment & $>0$ & $16(2)$ & $12(2)$ \\
B3LYP-D3 & 1.6 & -5 & -10 \\
B2PLYP-D3 & 2.2 & 1 & -4 \\
$\omega$ B97X-D & -0.04 & 7 & 3 \\
M06-2X & -2.2 & 29 & 24
\end{tabular}

$3561 \mathrm{~cm}^{-1}$ should not be completely dismissed at this stage. In any case B3LYP-D3 appears to consistently underestimate the hydrogen bond shift for cooperative $\mathrm{OH} \cdots \pi$ situations, whereas it overestimates them for isolated $\mathrm{OH} \cdots \pi$ and much more so for $\mathrm{OH} \cdots \mathrm{O}$ hydrogen bonds. While part of the latter overestimate is clearly due to a DFT deficiency, ${ }^{75}$ it may also be partly rooted in the harmonic approximation. Librational zeropoint energy generated by the directional interaction weakens the $\mathrm{OH} \cdots \mathrm{OH}$ hydrogen bond and the released strain may strengthen the neighboring $\mathrm{OH} \cdots \pi$ contact. However, this must remain speculative in the absence of high quality anharmonic calculations. In any case, the counterpropagating errors for isolated and cooperative $\mathrm{OH} \cdots \pi$ hydrogen bonds actually lead to a wrong sequence of bands at B3LYP-D3 level (Fig. 8), which in part persists for B2PLYP-D3 (Table 3). M06-2X and $\omega \mathrm{B} 97 \mathrm{X}-\mathrm{D}$ predict the correct sequence of bands, but perhaps for the wrong reason. This is supported by the wrong energy sequence prediction of $\omega \mathrm{B} 97 \mathrm{X}-\mathrm{D}$ and in particular M06-2X for the different hydrogen bond topologies. The experimental spectral intensities are not consistent with the prediction of a structure as the global minimum. The overestimated stability of $\mathrm{OH} \cdots \pi$ hydrogen bonds is reminiscent of the failure of M06-2X for the methanol/anisole complex. ${ }^{76}$

The comparison between theory and experiment can be extended to abundance estimates for the different isomers, once computed relative absorption band strengths and scattering cross sections are employed. By looking at band strength or cross section ratios for the two $\mathrm{OH}$ groups, some of the theoretical errors should cancel and by analyzing both IR and Raman intensities, the robustness of the abundance estimates can be checked. Table 4 lists the resulting intensity ratios between $\mathrm{OH} \cdots \mathrm{O}$ and $\mathrm{OH} \cdots \pi$ bands of homO ${ }^{g} \pi$.

One can see that in both experiment and theory, the IR ratio is somewhat larger than the Raman ratio. However, the IR ratio is overestimated by all methods but M06-2X. Due to the lower intensity and partial spectral overlap for the bands of het $O^{g} \pi$ we can only qualitatively state that their $\mathrm{O} / \pi$ ratio is likely larger than unity with both IR and Raman detection, in agreement with the predictions.

The experimental data from this work is used for up to four independent $T_{\mathrm{c}}$ estimates, using the $\mathrm{OH} \cdots \pi$ and $\mathrm{OH} \cdots \mathrm{O}$ band ratios as well as the IR and Raman spectra (see also Fig. S10 and S11 in the ESI†). Comparing bands of the same type (acceptor and detection technique) for both isomers allows for compensation of errors in absolute band strength predictions. The calculated 
Table 4 B dimer hom ${ }^{g} \pi \mathrm{OH}$ intensity ratios between $\mathrm{OH} \cdots \mathrm{O}$ and $\mathrm{OH} \cdots \pi$ bands from IR and Raman spectroscopy and from harmonic calculations, where available

\begin{tabular}{lll}
\hline Method & IR & Raman \\
\hline Experiment & $1.3(1)$ & $1.0(1)$ \\
B3LYP-D3 & 2.0 & 1.1 \\
B2PLYP-D3 & 2.1 & - \\
$\omega$ B97X-D & 2.2 & 1.1 \\
M06-2X & 1.4 & 1.2 \\
\hline
\end{tabular}

conformational temperature is therefore dominantly a representation of the predicted energy difference. All obtained $T_{\mathrm{c}}$ values for the interconversion between hetO $^{g} \pi$ and homo $^{g} \pi$ are positive, reflecting the robust qualitative prediction of the energy sequence. It is rewarding that the respective mean values do not vary strongly among the four estimates for each method, supporting both the spectral assignments and the assumptions behind the estimates. The B3LYP-D3 values $(12-23 \mathrm{~K})$ fall near the edge $(20 \mathrm{~K})$ of the plausible range, the B2PLYP-D3 ones (7-13 K) somewhat below it. This likely results from slight underestimations of the energy difference by these methods. The M06-2X temperatures are elevated (140-260 K) and represent in the absence of an indication for a high-barrier situation (confirmed at this level of theory) an overestimated energy difference. The $\omega$ B97X-D energy difference of $1.7 \mathrm{~kJ} \mathrm{~mol}^{-1}$ is likely the best estimate of these four methods, as it provides conformational freezing temperatures of 85-160 K, near the upper edge $(100 \mathrm{~K})$ expected for a low-barrier interconversion in a supersonic expansion.

Somewhat more speculatively, one can determine conformational freezing temperatures between $\operatorname{homo}^{g} \pi$ and het $\pi \pi$. Due to the different hydrogen bond topology, systematic error compensation for the cross sections may not work as well. By choosing the $\mathrm{OH} \cdots \pi$ - rather than the $\mathrm{OH} \cdots \mathrm{O}$-intensity of homO $^{g} \pi$, one might expect a better cancellation of cross section errors due to the analogous acceptor moiety. More critical is a potential contribution from hom $\pi \pi$ underneath the het $\pi \pi$ signal. This would generate $T_{\mathrm{c}}$ values which are too high, but so does the kinetic hindrance due to the high predicted barrier for het $\pi \pi$ relaxation. Therefore, conformational temperatures above $100 \mathrm{~K}$ but below the nozzle temperature are expected. At a temperature in this range, the collision-induced interconversion between the two isomers in the jet expansion comes to a halt. Obtained B3LYP-D3 and B2PLYP-D3 freezing temperatures are realistic $(100-230 \mathrm{~K})$, whereas the $\omega \mathrm{B} 97 \mathrm{X}-\mathrm{D}$ and M06-2X predictions are negative and thus unplausible. IR and Raman values are again nicely consistent.

In summary, the description of B dimers with DFT methods within the harmonic approximation is challenging. All four tested methods show different deficiencies for relative energies, band positions and spectral visibility. It is therefore imperative to verify this interpretation by a comparison with the related, permanently chiral P system, which allows for control of the hom/het ratio.

3.2.2 Experimental results for 1-phenylethanol. Fig. 9 provides a survey over the $\mathrm{OH}$ stretching range from 3670 to $3240 \mathrm{~cm}^{-1}$ for $\mathbf{P}$ expansions in helium. The IR spectra were

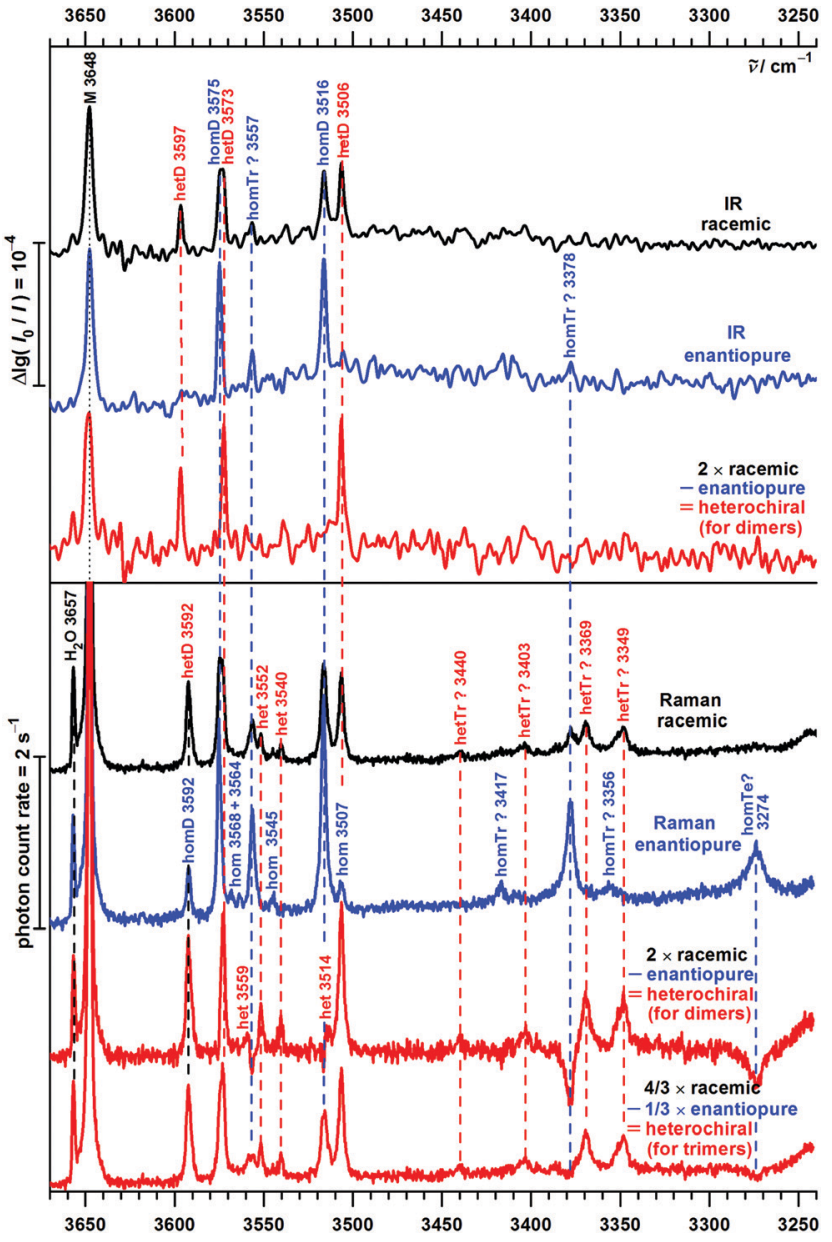

Fig. 9 Experimental FTIR (top) and Raman (bottom) spectra of 1-phenylethanol $\mathbf{P}$ in the $\mathrm{OH}$ stretching range. The concentration in the Raman detected expansion is considerably higher. Bands are marked with the relative chirality of the monomers, in parts with the probable cluster size ( $M=$ monomer, $\mathrm{D}=$ dimers, $\mathrm{Tr}=$ trimers, $\mathrm{Te}=$ tetramers $)$ and with their band position in $\mathrm{cm}^{-1}$

recorded with the long slit nozzle at room temperature, ${ }^{18}$ the Raman spectra required some heating and are therefore somewhat warmer and more oligomer-rich. Enantiopure and racemic samples were expanded and artificial spectra of heterochiral clusters can be generated by linear combinations. ${ }^{66}$ For dimers, $I_{\text {het }}^{\mathrm{D}}(\tilde{\nu})=2 I_{\text {rac }}(\tilde{\nu})-I_{\text {hom }}(\tilde{\nu})$ and for trimers $I_{\text {het }}^{\operatorname{Tr}}(\tilde{\nu})=$ $\frac{4}{3} I_{\mathrm{rac}}(\tilde{\nu})-\frac{1}{3} I_{\mathrm{hom}}(\tilde{\nu})$.

The sole monomer signal of $\mathbf{P}$ is found at $3648 \mathrm{~cm}^{-1}-$ the same position as for $\mathbf{B}$. This coincidence is partly due to a compensation of an expected downshift when switching from a primary to a secondary alcohol, and an expected upshift from a weakened intramolecular $\mathrm{OH} \cdots \pi$-interaction due to the lowering of the $\mathrm{OC}_{\alpha} \mathrm{C}_{i p s o} \mathrm{C}_{\text {ortho }}$ dihedral. The absence of other high frequency signals for both $\mathbf{B}$ and $\mathbf{P}$ is consistent with the high energy predictions for other monomer conformers as well as for dimers with one hydroxy group not acting as a donor in a hydrogen bond.

The FTIR spectra of enantiopure $\mathbf{P}$ contain in addition two intense bands at 3575 and $3516 \mathrm{~cm}^{-1}$. Their hom dimer 
character is supported by the statistical $50 \%$ intensity reduction in the spectrum of the racemic substance due to collision of monomers of the same chirality being half as likely. Assignment to homo ${ }^{g} \pi$ is straightforward by analogy to B. A weaker band at $3557 \mathrm{~cm}^{-1}$ is also reduced in the racemic expansion, but the reduction factor is difficult to determine due to the noise level. The Raman spectrum indicates a reduction factor larger than two and thus supports a trimer assignment, as does an analogous trimer band for $\mathbf{B}$ at $3561 \mathrm{~cm}^{-1}$. More downshifted oligomer Raman transitions are easily recognized by negative spectral contributions in the heterodimer subtraction procedure but are beyond the scope of this dimer study.

The somewhat broader band near $3574 \mathrm{~cm}^{-1}$ in the racemic spectra consists of closely spaced hom and het contributions, as the difference spectra reveal. Together with an intense band at $3506 \mathrm{~cm}^{-1}$ it clearly belongs to hetO ${ }^{g} \pi$, the het counterpart of the dominant hom dimer. This is supported by the $\mathbf{B}$ analogy and by the computations. In the range $3597-3592 \mathrm{~cm}^{-1}$, the heterochiral IR and Raman spectra are again complementary, thus reflecting the het $\pi \pi$ complex. Interestingly, the enantiopure Raman spectrum reveals a weaker band in the same position, which is the first positive evidence for hom $\pi \pi$ in this work. The IR counterpart is not observered, which may be explained by a poorer signal-to-noise ratio due to the high dilution and a tentatively colder expansion for the IR spectrum.

The warmer and more concentrated Raman detected expansion also reveals some weak bands (homochiral at 3568, 3564, 3545 and $3507 \mathrm{~cm}^{-1}$, heterochiral at 3559, 3552, 3540 and $3514 \mathrm{~cm}^{-1}$ ) which could be due to higher energy dimers, such as the $\mathrm{O}^{t} \pi$ motif (predicted to be more competitive in $\mathbf{P}$ than in B dimers), or cooperatively shifted trimer $\mathrm{OH} \cdots \pi$ interactions. Like more strongly shifted oligomer bands (homochiral at $3417,3378,3356$ and $3274 \mathrm{~cm}^{-1}$, heterochiral at 3440, 3403, 3369 and $3349 \mathrm{~cm}^{-1}$ ), which point at substantial oligomer chirality recognition phenomena reminiscent of the methyl lactate case,${ }^{5}$ they shall not be discussed further in the present dimer-focused work. We only note the close correspondence of the intense enantiopure $3378 \mathrm{~cm}^{-1}$ band for $\mathbf{P}$ with the $\mathbf{B}$ band at $3374 \mathrm{~cm}^{-1}$ (Fig. 7), which may point towards homochiral trimer chirality synchronization in $\mathbf{B}$ trimers. Whether this involves three-fold symmetry has to remain open.

A detailed comparison of experimental and simulated spectra in the dimer range is provided in Fig. 10.

The homo ${ }^{g} \pi$ and hetO $^{g} \pi$ assignments have already been discussed and are obvious. They confirm over- and underestimation trends for $\mathbf{B}$ and suggest that for the uncertain assignment of the $\mathbf{B}$ hetO ${ }^{g} \pi \mathrm{OH} \cdots \pi$ transition, the $3576 \mathrm{~cm}^{-1}$ band is more likely. For the $\pi \pi$ motif, a partial crossover of the harmonic predictions relative to $\mathrm{O}^{g} \pi$ is again found. The Davydov splitting of $\mathbf{P}$ het $\pi$ of $5 \mathrm{~cm}^{-1}$ is, as for $\mathbf{B}$, matched by all four quantum chemical methods. However, it is unexpected by all four harmonic methods that the symmetric $\mathrm{OH}$ stretching vibrations of het $\pi \pi$ and hom $\pi \pi$ are coinciding. The smallest mismatch is predicted by B2PLYP-D3 $\left(\Delta \omega=4 \mathrm{~cm}^{-1}\right)$. The hom - het chirality discrimination shifts for the three observed band pairs are summarized in Table 5 .

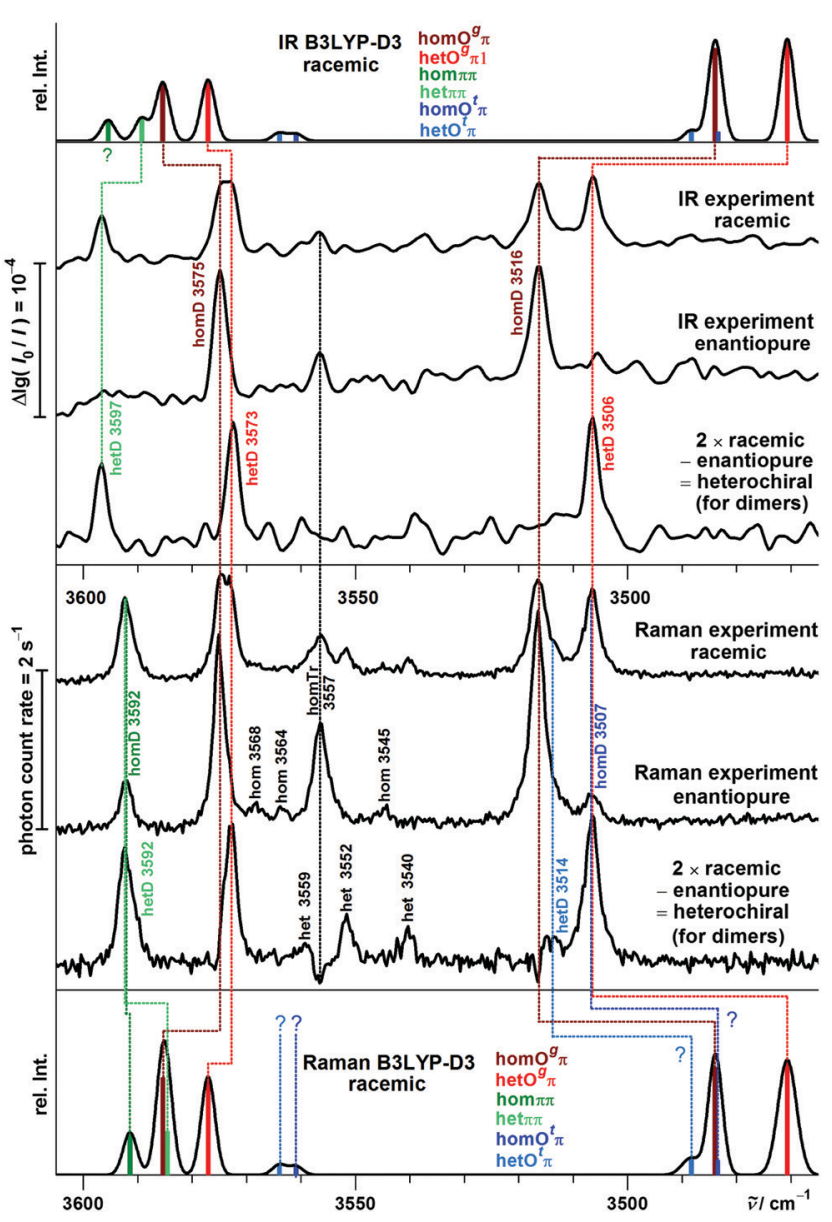

Fig. $10 \mathbf{P}$ dimer B3LYP-D3 simulations (outer, racemic only, separate equilibria for hom and het) compared to experimental IR and Raman jet spectra (inner traces) after shifting the harmonic $\mathrm{OH}$ stretching wavenumbers by $-165.2 \mathrm{~cm}^{-1}$ to match the monomer transition. A uniform conformational temperature of $100 \mathrm{~K}$ and a Gaussian FWHM of $3 \mathrm{~cm}^{-1}$ are assumed.

Table 5 Comparison between experiment and harmonic theory for $\mathrm{OH}$ stretching shifts between $\mathbf{P}$ dimers (hom - het) upon change of relative chirality in $\mathrm{cm}^{-1}$

\begin{tabular}{lccc}
\hline Method & $\pi \pi$ symm. & $\mathrm{O}^{g} \pi \mathrm{OH} \cdots \pi$ & $\mathrm{O}^{g} \pi \mathrm{OH} \cdots \mathrm{O}$ \\
\hline Experiment & $0(2)$ & $2(2)$ & $10(2)$ \\
B3LYP-D3 & 7 & 8 & 13 \\
B2PLYP-D3 & 4 & 9 & 12 \\
$\omega$ B97X-D & 9 & 13 & 10 \\
M06-2X & -11 & 4 & 9
\end{tabular}

Fig. 10 also includes simulation contributions from $\mathrm{O}^{t} \pi$ structures, which are energetically somewhat lowered for $\mathbf{P}$ relative to $\mathbf{B}$. These contribute at best minor signals and shoulders and shall therefore not be discussed further.

In analogy to $\mathbf{B}$, conformational freezing temperatures can be estimated for the hydrogen bond topology switch, now separately for hom and het pairings (see also Fig. S12 and S13 in the ESI $\dagger$ ). The expectation is that they reflect the higher barrier in the het $\pi \pi$ case. Without positive IR evidence of hom $\pi \pi$, only an upper bound from the FTIR data is possible: 


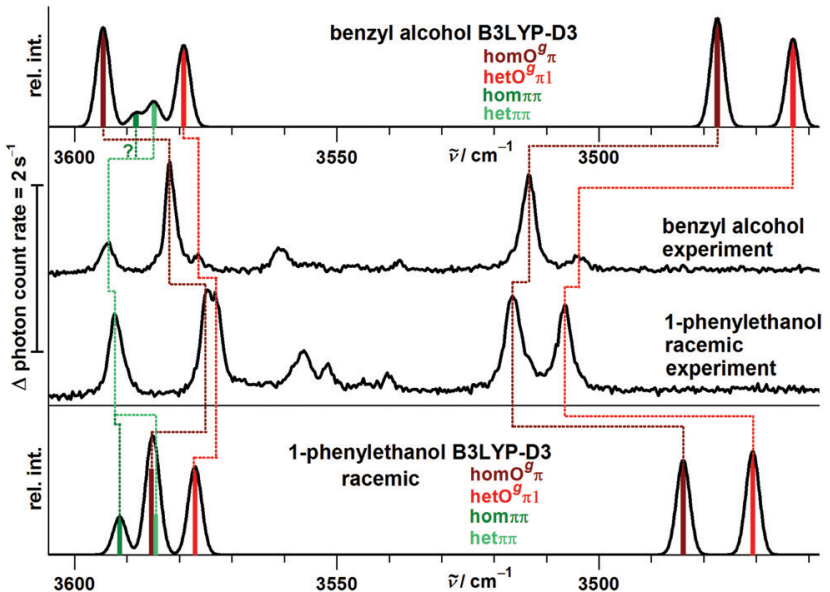

Fig. 11 Simulated and experimental Raman spectra for $\mathbf{B}$ and $\mathbf{P}$ dimers after uniform wavenumber shift to reproduce the respective monomer $\mathrm{OH}$ stretch, using a uniform conformational temperature of $100 \mathrm{~K}$ and $3 \mathrm{~cm}^{-1}$ FWHM Gaussian profiles.

$<(70-130) \mathrm{K}$ for B3LYP-D3, B2YPLYP-D3 and $\omega$ B97X-D, but negative for M06-2X. The temperatures obtained from the Raman spectrum for the homochiral case are realistic for a low-barrier interconversion with B3LYP-D3 and $\omega$ B97X-D predictions (60-100 K) but are strongly negative for M06-2X, due to the wrong prediction of the global minimum structure. For the relaxation of het $\pi \pi$ the B3LYP-D3 und B2PLYP-D3 predictions are significantly higher $(200-400 \mathrm{~K})$, as one would expect for kinetic hindrance. The values for $\omega$ B97X-D and M06-2X are again strongly negative. These results are similar to the analogous situation for $\mathbf{B}$.

The close analogy between $\mathbf{B}$ and $\mathbf{P}$ and the regularity in the harmonic prediction failure of band centers is summarized in Fig. 11 for the Raman spectra, focusing on the four most stable conformations. Double methylation of $\mathbf{B}$ dimers has a slight weakening effect on the $\mathrm{OH} \cdots \mathrm{O}$ hydrogen bond which translates into a formal strengthening effect for the cooperatively
Table $6 \mathbf{P}$ dimer intensity ratios of $\mathrm{OH} \cdots \mathrm{O}$ and $\mathrm{OH} \cdots \pi$ modes for $\mathrm{IR}$ and Raman detection by experiment and harmonic theory

\begin{tabular}{|c|c|c|c|c|}
\hline \multirow[b]{2}{*}{ Method } & \multicolumn{2}{|c|}{$\operatorname{homO}^{g} \pi$} & \multicolumn{2}{|c|}{$\operatorname{hetO}^{g} \pi$} \\
\hline & IR & Raman & IR & Raman \\
\hline Experiment & $0.9(2)$ & $1.5(2)$ & $0.9(5)$ & $1.1(3)$ \\
\hline B3LYP-D3 & 1.6 & 1.1 & 1.7 & 1.1 \\
\hline B2PLYP-D3 & 1.5 & - & 1.6 & - \\
\hline$\omega \mathrm{B} 97 \mathrm{X}-\mathrm{D}$ & 1.7 & 1.1 & 1.7 & 1.3 \\
\hline M06-2X & 1.1 & 1.3 & 1.3 & 1.4 \\
\hline
\end{tabular}

attached $\mathrm{OH} \cdots \pi$ hydrogen bond. As mentioned before, this slight formal anti-cooperativity might have a partially anharmonic origin, but in any case it is qualitatively reproduced at harmonic B3LYP-D3 level, as is the slight downshift of the het $\pi$ band.

Coming back to the intensity ratio between the $\mathrm{O}$ and $\pi$ acceptor hydrogen bonds in the $\mathrm{O}^{g} \pi$ motif, which was predicted slightly anomalously in the harmonic DFT calculations for $\mathbf{B}$, the corresponding $\mathbf{P}$ results are summarized in Table 6. Similar to B (Table 4), B3LYP-D3, B2PLYP-D3 and $\omega$ B97X-D predict a higher $\mathrm{O}$ acceptor visibility in the IR and a similar visibility in the Raman spectra. Experimentally, the opposite is observed for $\mathbf{P}$. This distinction between $\mathbf{B}$ and $\mathbf{P}$ can be related to a smaller wavenumber difference between the transitions and thus a stronger coupling and redistribution of IR and Raman activity in $\mathbf{P}$, which is sensitive to accurate hydrogen bond shifts. While this trend in wavenumber difference is predicted by all four methods, only M06-2X predicts the actual values (Table 7) and thus the band intensity ratios (Table 6) almost within the respective experimental uncertainties.

Whether this is for the right reason or due to error compensation between deficiencies in the electronic structure method (overestimation of the relative strength of the $\mathrm{OH} \cdots \pi$ hydrogen bond) and the double-harmonic approximation, has to remain an open question at this time. An interesting test case could be the dimers of propargyl alcohol, for which two conformers were

\begin{tabular}{|c|c|c|c|c|c|}
\hline Property & B3LYP-D3 & B2PLYP-D3 & $\omega \mathrm{B} 97 \mathrm{X}-\mathrm{D}$ & M06-2X & Source \\
\hline Energy: $\mathrm{B} \mathrm{O}^{g} \pi$ hom/het preference & & & & & Fig. S10 \\
\hline Energy: $\mathrm{B}$ homo ${ }^{g} \pi /$ het $\pi \pi$ preference & & & & & Fig. S11 \\
\hline Energy: $\mathbf{P}$ homO ${ }^{g} \pi /$ hom $\pi \pi$ preference & & & & & Fig. S12 \\
\hline Energy: $\mathbf{P}$ het ${ }^{g} \pi /$ het $\pi \pi$ preference & & & & & Fig. S13 \\
\hline Wavenumber: B \& $\mathbf{P}$ het $\pi \pi$ Davydov splittings & & & & & Tab. 1 \\
\hline Wavenumber: $\mathbf{B}$ homO ${ }^{g} \pi /$ het $\pi \pi$ differences & & & & & Tab. 3 \\
\hline Wavenumber: $\mathbf{B} \mathrm{O}^{g} \pi$ hom/het differences & & & & & Tab. 2 \\
\hline Wavenumber: $\mathbf{P} \mathrm{O}^{g} \pi$ hom/het differences & & & & & Tab. 5 \\
\hline Wavenumber: $\mathbf{P} \pi \pi$ hom/het differences & & & & & Tab. 5 \\
\hline Wavenumber: B \& $\mathbf{P}$ homO $^{g} \pi$ acceptor differences & & & & & Tab. 7 \\
\hline Visibility: B homO ${ }^{g} \pi \mathrm{IR} /$ Raman trend & & & & & Tab. 4 \\
\hline Visibility: P hom- \& hetO ${ }^{g} \pi \mathrm{IR} /$ Raman trend & & & & & Tab. 6 \\
\hline
\end{tabular}

Fig. 12 Rating of harmonic quantum chemical methods for $\mathbf{B}$ and $\mathbf{P}$ experimental dimer results presented in tables and figures in this work, in the categories energy, wavenumber and spectral visibility performance. Quantitative agreement with experiment within uncertainties is marked in green, qualitative agreement in yellow, and qualitative disagreement in red. No color means non-coverage due to unaffordable Raman activities for B2PLYP-D3. 
Table $7 \quad \mathbf{B}$ and $\mathbf{P} \mathrm{OH}$ stretching wavenumber differences $\Delta \tilde{\nu}(\omega)$ between the $\mathrm{OH} \cdots \pi$ and the $\mathrm{OH} \cdots \mathrm{O}$ modes of hom $\mathrm{O}^{g} \pi$ at different harmonic levels in comparison with experiment

\begin{tabular}{lcc}
\hline Method & $\Delta \tilde{\nu}(\omega) \mathbf{B} / \mathrm{cm}^{-1}$ & $\Delta \tilde{\nu}(\omega) \mathbf{P} / \mathrm{cm}^{-1}$ \\
\hline Experiment & $68(2)$ & $59(2)$ \\
B3LYP-D3 & 117 & 102 \\
B2PLYP-D3 & 106 & 88 \\
WB97X-D & 115 & 103 \\
M06-2X & 69 & 62
\end{tabular}

recently assigned in an FTIR matrix study. ${ }^{64}$ In the nomenclature used here they correspond to homo ${ }^{t} \pi$ and homo ${ }^{g} \pi$ structures, with the former having weakly and the latter strongly coupled (assigned only $20 \mathrm{~cm}^{-1}$ apart) OH stretching modes.

\section{Conclusions}

This work can be summarized on a puzzle-solving and on an experimental benchmarking level.

On the puzzle-solving level, the close analogy between benzyl alcohol and 1-phenylethanol dimerization together with an extensive characterization of the potential energy landscape has secured and explained three previous chirality-sensitive conjectures and open assignments for the dimer of $\mathbf{B}$ :

- The global minimum structure of $\mathbf{B}$ dimer is confirmed to involve homochirally synchronized monomers.

- The heterochiral B dimer counterpart is now spectrally assigned and its relative energy is about $0.3-2.0 \mathrm{~kJ} \mathrm{~mol}^{-1}$ higher.

- The dominant $\pi \pi$ dimer is almost certainly heterochiral, because the $C_{\mathrm{i}}$ symmetry generates a higher trapping barrier, whereas the homochiral counterpart relaxes efficiently.

These conclusions are largely experimental, but they profit from reliable computational assignment aids. For systems of this size, these computations typically have to employ the harmonic approximation. Therefore, a formally good agreement between theory and experiment could be right for the wrong reason and if it is non-systematic, it is not so helpful to the spectroscopist. Systematic deviations, such as overestimations of certain hydrogen bond-induced spectral shifts and underestimations for other types of hydrogen bonds due to anharmonicity, are more acceptable, in particular once they are well understood. A difficult task ahead is to understand why certain hydrogen bond patterns have systematically different anharmonicities. This work provides ample testing ground for such an analysis.

On an experimental benchmarking level, the final assignment of the FTIR and Raman spectra allows for statements about the relative energy of different dimer conformations. These can be directly compared to harmonically zero-point energy-corrected predictions for the four investigated methods. Their performance is summarized in Fig. 12. Because of the two phenyl rings involved, a proper description of London dispersion interactions is essential for the correct energy balance of the different dimer conformations. At least when combined with the may-cc-pVTZ basis set and the double-harmonic approximation, none of the benchmarked functionals strikes this balance in a fully satisfactory way within plausible experimental range, with B3LYP-D3 coming closest. For spectral assignment purposes predicted wavenumber differences suffer from the over- and underestimation of shifts for different hydrogen bonds, ${ }^{74}$ which might be partly blamed on the harmonic approximation. ${ }^{75}$ This also results in unreliable visibility ratios already for weakly coupled $\mathrm{OH}$ stretching modes, as indicated by results of this work. Predictions for the investigated systems by the double-hybrid B2PLYP-D3 functional (as far as accessible) are overall very similar to B3LYP-D3 and are apparently not profiting from the included high-cost perturbative second-order correlation part. $\omega$ B97X-D slightly and M06-2X strongly predicts wrong global minima. However, the latter functional shows the best overall predictions for wavenumber differences and related relative spectral visibility, partly because it does not share the generic overestimation of large hydrogen bond-induced shifts of most DFT methods. This is reminiscent of a broader benchmark for hydrogen bonding. ${ }^{77}$ It would be rewarding to have a functional which makes better shift predictions for hydrogen bonds without losing predictive power for the energetics of competing isomers.

The dimerization picture which emerges for benzyl alcohol in this work is somewhat analogous but also opposite to that of ethanediol, ${ }^{6}$ beyond the fact that the chirality is much more transient for the former. Homochiral encounters are funneled down rather efficiently to the global minimum structure of benzyl alcohol, whereas this is closer to reality for heterochiral encounters in ethanediol. A significant fraction of heterochiral encounters gets trapped behind barriers for benzyl alcohol, whereas this is strongly the case for homochiral encounters for ethanediol. For benzyl alcohol, there is the potential for a detailed investigation of such processes by stimulated emission pumping, ${ }^{78}$ because it offers an aromatic chromophore. It would be interesting to test our conjectures using this or other powerful techniques, because experimental benchmarks need experimental verification.

\section{Conflicts of interest}

There are no conflicts to declare.

\section{Acknowledgements}

This project was partly funded by the Deutsche Forschungsgemeinschaft (DFG, German Research Foundation) - 271107160/ SPP1807. Computational ressources from the GWDG and the Faculty of Chemistry (DFG - 405832858/INST 186/1294-1 FUGG) are acknowledged. The benchmarking aspect has profited from the environment provided by the local research training group BENCh (DFG - 389479699/GRK2455). We thank E. Meyer and C. Stelbrink for help in the context of their undergraduate research participation and the workshops of the institute for 
their valuable support. M. Lange and E. Sennert have kindly provided an improved benzyl alcohol IR jet spectrum. M. Gawrilow has provided instrumental support. N. O. B. Lüttschwager has provided the MC integration method and support for it.

\section{Notes and references}

1 L.-C. Sögütoglu, R. R. E. Steendam, H. Meekes, E. Vlieg and F. P. J. T. Rutjes, Chem. Soc. Rev., 2015, 44, 6723-6732.

2 A. Kumar, E. Capua, M. K. Kesharwani, J. M. L. Martin, E. Sitbon, D. H. Waldeck and R. Naaman, Proc. Natl. Acad. Sci. U. S. A., 2017, 114, 2474-2478.

3 C. Tschierske and G. Ungar, ChemPhysChem, 2016, 17, 9-26. 4 J. Jacques, A. Collet and S. H. Wilen, Enantiomers, racemates, and resolutions, Krieger, Malabar, Florida, Reissue with corrections, 1994.

5 A. Zehnacker and M. A. Suhm, Angew. Chem., Int. Ed., 2008, 47, 6970-6992.

6 B. Hartwig, M. Lange, A. Poblotzki, R. Medel, A. Zehnacker and M. A. Suhm, Phys. Chem. Chem. Phys., 2020, 22, 1122-1136.

7 S. Portmann, A. Inauen, H. P. Lüthi and S. Leutwyler, J. Chem. Phys., 2000, 113, 9577-9585.

8 T. N. Wassermann and M. A. Suhm, J. Phys. Chem. A, 2010, 114, 8223-8233.

9 D. Loru, I. Peña and M. Sanz, J. Mol. Spectrosc., 2017, 335, 93-101. 10 T. Scharge, T. Häber and M. A. Suhm, Phys. Chem. Chem. Phys., 2006, 8, 4664-4667.

11 J. Thomas and Y. Xu, J. Phys. Chem. Lett., 2014, 5, 1850-1855.

12 M. Mons, E. G. Robertson and J. P. Simons, J. Phys. Chem. A, 2000, 104, 1430-1437.

13 J. Altnöder, S. Oswald and M. A. Suhm, J. Phys. Chem. A, 2014, 118, 3266-3279.

14 J. C. Pearson, K. V. L. N. Sastry, E. Herbst and F. C. De Lucia, Astrophys. J., 1997, 480, 420-431.

15 J. J. Fox and A. E. Martin, Trans. Faraday Soc., 1940, 36, 897.

16 N. Guchhait, T. Ebata and N. Mikami, J. Chem. Phys., 1999, 111, 8438-8447.

17 N. Guchhait, T. Ebata and N. Mikami, J. Am. Chem. Soc., 1999, 121, 5705-5711.

18 M. A. Suhm and F. Kollipost, Phys. Chem. Chem. Phys., 2013, 15, 10702.

19 S. Oswald, Aromatische und aliphatische Sechsringe in Wasserstoffbrückenkonkurrenz, Bachelor's thesis, GeorgAugust-Universität Göttingen, 2013, unpublished.

20 N. A. Seifert, A. L. Steber, J. L. Neill, C. Pérez, D. P. Zaleski, B. H. Pate and A. Lesarri, Phys. Chem. Chem. Phys., 2013, 15, 11468.

21 M. Mons, F. Piuzzi, I. Dimicoli, A. Zehnacker and F. Lahmani, Phys. Chem. Chem. Phys., 2000, 2, 5065-5070.

22 A. Giardini Guidoni, S. Piccirillo, D. Scuderi, M. Satta, T. M. Di Palma and M. Speranza, Phys. Chem. Chem. Phys., 2000, 2, 4139-4142.

23 A. Giardini Guidoni, S. Piccirillo, D. Scuderi, M. Satta, T. Di Palma, M. Speranza, A. Filippi and A. Paladini, Chirality, 2001, 13, 727-730.
24 K. Le Barbu, F. Lahmani, M. Mons, M. Broquier and A. Zehnacker, Phys. Chem. Chem. Phys., 2001, 3, 4684-4688.

25 K. Le Barbu, A. Zehnacker, F. Lahmani, M. Mons, F. Piuzzi and I. Dimicoli, Chirality, 2001, 13, 715-721.

26 A. Filippi, A. Giardini, A. Latini, S. Piccirillo, D. Scuderi and M. Speranza, Int. J. Mass Spectrom., 2001, 210-211, 483-488.

27 D. Scuderi, A. Paladini, M. Satta, D. Catone, A. Filippi, S. Piccirillo, A. Laganà, M. Speranza and A. Guidoni, Int. J. Mass Spectrom., 2003, 223-224, 159-168.

28 R. Medel, C. Stelbrink and M. A. Suhm, Angew. Chem., Int. Ed., 2019, 58, 8177-8181.

29 K. Shin-ya, H. Sugeta, S. Shin, Y. Hamada, Y. Katsumoto and K. Ohno, J. Phys. Chem. A, 2007, 111, 8598-8605.

30 F. Kollipost, K. E. Otto and M. A. Suhm, Angew. Chem., Int. Ed., 2016, 55, 4591-4595.

31 S. K. Nayak, R. Sathishkumar and T. N. G. Row, CrystEngComm, 2010, 12, 3112. Note that the position of the hydroxy hydrogen atom in the single molecule cutout in Fig. 4 of the reference is not in agreement with the crystal structure.

32 T. Forsting and M. Suhm, Curry-Jet SETUP, 2019, DOI: 10.6084/m9.figshare.6395840.v1.

33 M. J. Frisch, G. W. Trucks, H. B. Schlegel, G. E. Scuseria, M. A. Robb, J. R. Cheeseman, G. Scalmani, V. Barone, B. Mennucci, G. A. Petersson, H. Nakatsuji, M. Caricato, X. Li, H. P. Hratchian, A. F. Izmaylov, J. Bloino, G. Zheng, J. L. Sonnenberg, M. Hada, M. Ehara, K. Toyota, K. Fukuda, J. Hasegawa, M. Ishida, T. Nakajima, Y. Honda, O. Kitao, H. Nakai, T. Vreven, J. A. J. Montgomery, J. E. Peralta, F. Ogliaro, M. Bearpark, J. J. Heyd, E. Brothers, K. N. Kudin, V. N. Staroverov, R. Kobayashi, J. Normand, K. Raghavachari, A. Rendell, J. C. Burant, S. S. Iyengar, J. Tomasi, M. Cossi, N. Rega, J. M. Millam, M. Klene, J. E. Knox, J. B. Cross, V. Bakken, C. Adamo, J. Jaramillo, R. Gomperts, R. E. Stratmann, O. Yazyev, A. J. Austin, R. Cammi, C. Pomelli, J. W. Ochterski, R. L. Martin, K. Morokuma, V. G. Zakrzewski, G. A. Voth, P. Salvador, J. J. Dannenberg, S. Dapprich, A. D. Daniels, Ö. Farkas, J. B. Foresman, J. V. Ortiz, J. Cioslowski and D. J. Fox, Gaussian 09, Revision E.01, Gaussian, Inc. technical report, 2009.

34 A. D. Becke, Phys. Rev. A: At., Mol., Opt. Phys., 1988, 38, 3098-3100.

35 C. Lee, W. Yang and R. G. Parr, Phys. Rev. B: Condens. Matter Mater. Phys., 1988, 37, 785-789.

36 A. D. Becke, J. Chem. Phys., 1993, 98, 5648-5652.

37 P. J. Stephens, F. J. Devlin, C. F. Chabalowski and M. J. Frisch, J. Phys. Chem., 1994, 98, 11623-11627.

38 S. Grimme, S. Ehrlich and L. Goerigk, J. Comput. Chem., 2011, 32, 1456-1465.

39 F. Weigend and R. Ahlrichs, Phys. Chem. Chem. Phys., 2005, 7, 3297.

40 E. Papajak, J. Zheng, X. Xu, H. R. Leverentz and D. G. Truhlar, J. Chem. Theory Comput., 2011, 7, 3027-3034.

41 S. Grimme, J. Chem. Phys., 2006, 124, 034108.

42 J.-D. Chai and M. Head-Gordon, Phys. Chem. Chem. Phys., 2008, 10, 6615 . 
43 Y. Zhao and D. G. Truhlar, Theor. Chem. Acc., 2008, 120, 215-241.

44 G. Karir, N. O. B. Lüttschwager and M. A. Suhm, Phys. Chem. Chem. Phys., 2019, 21, 7831-7840.

45 A. Poblotzki, H. C. Gottschalk and M. A. Suhm, J. Phys. Chem. Lett., 2017, 8, 5656-5665.

46 H. C. Gottschalk, A. Poblotzki, M. A. Suhm, M. M. AlMogren, J. Antony, A. A. Auer, L. Baptista, D. M. Benoit, G. Bistoni, F. Bohle, R. Dahmani, D. Firaha, S. Grimme, A. Hansen, M. E. Harding, M. Hochlaf, C. Holzer, G. Jansen, W. Klopper, W. A. Kopp, L. C. Kröger, K. Leonhard, H. Mouhib, F. Neese, M. N. Pereira, I. S. Ulusoy, A. Wuttke and R. A. Mata, J. Chem. Phys., 2018, 148, 014301.

47 H. C. Gottschalk, A. Poblotzki, M. Fatima, D. A. Obenchain, C. Pérez, J. Antony, A. A. Auer, L. Baptista, D. M. Benoit, G. Bistoni, F. Bohle, R. Dahmani, D. Firaha, S. Grimme, A. Hansen, M. E. Harding, M. Hochlaf, C. Holzer, G. Jansen, W. Klopper, W. A. Kopp, M. Krasowska, L. C. Kröger, K. Leonhard, M. Mogren Al-Mogren, H. Mouhib, F. Neese, M. N. Pereira, M. Prakash, I. S. Ulusoy, R. A. Mata, M. A. Suhm and M. Schnell, J. Chem. Phys., 2020, 152, 164303.

48 R. Glaser and G. R. Nichols, J. Org. Chem., 2000, 65, 755-766.

49 V. V. Varfolomeeva and A. V. Terent'ev, Russ. J. Phys. Chem. A, 2010, 84, 1592-1597.

50 B. J. Miller, H. G. Kjaergaard, K. Hattori, S.-i. Ishiuchi and M. Fujii, Chem. Phys. Lett., 2008, 466, 21-26.

51 K. A. Utzat, R. K. Bohn, J. A. Montgomery, H. H. Michels and W. Caminati, J. Phys. Chem. A, 2010, 114, 6913-6916.

52 T. V. Alves, L. Simón-Carballido, F. R. Ornellas and A. FernándezRamos, Phys. Chem. Chem. Phys., 2016, 18, 8945-8953.

53 S. Tang, Z. Xia, A. Maris and W. Caminati, Chem. Phys. Lett., 2010, 498, 52-55.

54 L. Evangelisti, L. B. Favero and W. Caminati, J. Mol. Struct., 2010, 978, 279-281.

55 R. G. Bird, A. E. Nikolaev and D. W. Pratt, J. Phys. Chem. A, 2011, 115, 11369-11377.

56 L. Evangelisti, G. Feng, Q. Gou and W. Caminati, J. Mol. Struct., 2012, 1023, 15-17.

57 L. Evangelisti, Q. Gou, G. Feng and W. Caminati, Mol. Phys., 2013, 111, 1994-1998.

58 R. T. Saragi, M. Juanes, W. Caminati, A. Lesarri, L. Enríquez and M. Jaraíz, J. Phys. Chem. A, 2019, 123, 8435-8440.
59 B. Maté, R. D. Suenram and C. Lugez, J. Chem. Phys., 2000, 113, 192-199.

60 J. Kapitán, C. Johannessen, P. Bouř, L. Hecht and L. D. Barron, Chirality, 2009, 21, E4-E12.

61 E. Bogdan, G. Compain, L. Mtashobya, J.-Y. Le Questel, F. Besseau, N. Galland, B. Linclau and J. Graton, Chem. Eur. J., 2015, 21, 11462-11474.

62 A. K. King and B. J. Howard, J. Mol. Spectrosc., 2009, 257, 205-212.

63 D. Mani and E. Arunan, J. Chem. Phys., 2014, 141, 164311.

64 J. Saini and K. S. Viswanathan, J. Phys. Chem. A, 2017, 121, 1448-1459.

65 K. A. E. Meyer and M. A. Suhm, J. Chem. Phys., 2018, 149, 104307.

66 N. Borho and M. A. Suhm, Org. Biomol. Chem., 2003, 1, 4351.

67 N. Borho and M. A. Suhm, Phys. Chem. Chem. Phys., 2002, 4, 2721-2732.

68 J. Altnöder, J. J. Lee, K. E. Otto and M. A. Suhm, ChemistryOpen, 2012, 1, 269-275.

69 R. S. Ruoff, T. D. Klots, T. Emilsson and H. S. Gutowsky, J. Chem. Phys., 1990, 93, 3142-3150.

70 J. Altnöder, A. Bouchet, J. J. Lee, K. E. Otto, M. A. Suhm and A. Zehnacker-Rentien, Phys. Chem. Chem. Phys., 2013, 15, 10167.

71 J. A. Frey, C. Holzer, W. Klopper and S. Leutwyler, Chem. Rev., 2016, 116, 5614-5641.

72 S. Haldar, R. Gnanasekaran and P. Hobza, Phys. Chem. Chem. Phys., 2015, 17, 26645-26652.

73 E. Sennert, Methyllaktat als multifunktionaler chiraler Induktor, Bachelor's thesis, Georg-August-Universität Göttingen, 2019, unpublished.

74 R. Medel, M. Heger and M. A. Suhm, J. Phys. Chem. A, 2015, 119, 1723-1730.

75 M. Heger, M. A. Suhm and R. A. Mata, J. Chem. Phys., 2014, 141, 101105.

76 H. C. Gottschalk, J. Altnöder, M. Heger and M. A. Suhm, Angew. Chem., Int. Ed., 2016, 55, 1921-1924.

77 S. Oswald and M. A. Suhm, Phys. Chem. Chem. Phys., 2019, 21, 18799-18810.

78 B. C. Dian, J. R. Clarkson and T. S. Zwier, Science, 2004, 303, 1169-1173. 